Mon. Not. R. Astron. Soc. 000, 1, 23 (2015) Printed 3 October $2018 \quad$ (MN LTEX style file v2.2)

\title{
Estimating stellar atmospheric parameters, absolute magnitudes and elemental abundances from the LAMOST spectra with Kernel-based Principal Component Analysis
}

\author{
M.-S. Xiang ${ }^{1 \star} \dagger$, X.-W. Liu ${ }^{2,3}$, J.-R. Shi ${ }^{1}$, H.-B. Yuan ${ }^{4}$, Y. Huang ${ }^{2}$, A.-L. Luo ${ }^{1}$, \\ H.-W. Zhang ${ }^{2}$, Y.-H. Zhao ${ }^{1}$, J.-N. Zhang ${ }^{1}$, J.-J., Ren ${ }^{2 \star}$, B.-Q. Chen ${ }^{2 \star}$, C. Wang ${ }^{2}$, \\ J. Li ${ }^{5}$, Z.-Y. Huo ${ }^{1}$, W. Zhang ${ }^{1}$, J.-L. Wang ${ }^{1}$, Y. Zhang ${ }^{6}$, Y.-H. Hou ${ }^{6}$, Y.-F. Wang ${ }^{6}$ \\ 1 National Astronomical Observatories, Chinese Academy of Sciences, Beijing 100012, P. R. China \\ 2 Department of Astronomy, Peking University, Beijing 100871, P. R. China \\ 3 Kavli Institute for Astronomy and Astrophysics, Peking University, Beijing 100871, P. R. China \\ 4 Department of Astronomy, Beijing Normal University, Beijing 100875, P. R. China \\ 5 Department of Space Science and Astronomy, Hebei Normal University, Shijiazhuang 050024, P. R. China \\ 6 Nanjing Institute of Astronomical Optics \& Technology, National Astronomical Observatories, Chinese Academy of Sciences, Nanjing 210042, P. R. China
}

Received:

\begin{abstract}
Accurate determination of stellar atmospheric parameters and elemental abundances is crucial for Galactic archeology via large-scale spectroscopic surveys. In this paper, we estimate stellar atmospheric parameters - effective temperature $T_{\text {eff }}$, surface gravity $\log g$ and metallicity $[\mathrm{Fe} / \mathrm{H}]$, absolute magnitudes $\mathrm{M}_{V}$ and $\mathrm{M}_{K \mathrm{~s}}, \alpha$-element to metal (and iron) abundance ratio $[\alpha / \mathrm{M}]$ (and $[\alpha / \mathrm{Fe}]$ ), as well as carbon and nitrogen abundances $[\mathrm{C} / \mathrm{H}]$ and $[\mathrm{N} / \mathrm{H}]$ from the LAMOST spectra with a multivariate regression method based on kernel-based principal component analysis, using stars in common with other surveys (Hipparcos, Kepler, APOGEE) as training data sets. Both internal and external examinations indicate that given a spectral signal-to-noise ratio (SNR) better than 50, our method is capable of delivering stellar parameters with a precision of $\sim 100 \mathrm{~K}$ for $T_{\text {eff }}, \sim 0.1$ dex for $\log g, 0.3-0.4 \mathrm{mag}$ for $\mathrm{M}_{V}$ and $\mathrm{M}_{K \mathrm{~s}}, 0.1 \mathrm{dex}$ for $[\mathrm{Fe} / \mathrm{H}],[\mathrm{C} / \mathrm{H}]$ and $[\mathrm{N} / \mathrm{H}]$, and better than 0.05 dex for $[\alpha / \mathrm{M}]([\alpha / \mathrm{Fe}])$. The results are satisfactory even for a spectral SNR of 20. The work presents first determinations of $[\mathrm{C} / \mathrm{H}]$ and $[\mathrm{N} / \mathrm{H}]$ abundances from a vast data set of LAMOST, and, to our knowledge, the first reported implementation of absolute magnitude estimation directly based on the observed spectra. The derived stellar parameters for millions of stars from the LAMOST surveys will be publicly available in the form of value-added catalogues.
\end{abstract}

Key words: Galaxy: evolution - stars: abundance - stars: fundamental parameters - techniques: spectroscopic

\section{INTRODUCTION}

Accurate estimation of stellar atmospheric parameters and elemental abundances from large samples of high-to-low resolution spectra collected by modern large spectroscopic surveys, such as the LAMOST Experiment for Galactic Understanding and Exploration (LEGUE; Deng et al. 2012; Zhao et al. 2012), the Sloan Extension for Galactic Understanding and Exploration (SEGUE; Yanny et al. 2009), the Apache Point Observatory Galactic Evolution Experiment (APOGEE Majewski et al. 2010), the

* LAMOST Fellow

† E-mail: msxiang@nao.cas.cn
Radial Velocity Experiment (RAVE; Steinmetz et al. 2006), the High Efficiency and Resolution Multi-Element Spectrograph survey (HERMES; Zucker et al. 2012; Freeman 2012), and the Gaia Radial Velocity Spectrograph (RVS) survey (e.g. Bailer-Jones et al. 2013), is of vital importance for Galactic archaeology. For this purpose, various methods have been developed, based on either a $\chi^{2}$-related algorithm (Allende Prieto et al. 2006; Lee et al. 2008a; Zwitter et al. 2008; Wu et al. 2011, 2014; Xiang et al. 2015a; García Pérez et al. 2016) or a multivariable analysis (e.g. principal component analysis, PCA) incorporated into a neural network scheme (Recio-Blanco, Bijaoui \& de Laverny 2006; Re Fiorentin et al. 2007; Manteiga et al. 2010; Yang \& Li 2015; 
Lu \& Li 2015; Li et al. 2015; Bu \& Pan 2015; Liu et al. 2015; Recio-Blanco et al. 2016).

In spite of the efforts, in the case of low-resolution spectra, the accuracy remains to be improved, especially for parameter such as the surface gravity $\log g$ that has a relatively weak dependence on the observed spectral features. Typical uncertainties of $\log g$ estimates yielded by most pipelines are about $0.2 \mathrm{dex}$ or larger (Lee et al. 2008a b; Zwitter et al. 2008; Kordopatis et al. 2013; Xiang et al. 2015a; Gao et al. 2015). Patterns of systematic errors in $\log g$ are clearly visible in the results of SEGUE Stellar Parameter Pipeline (SSPP) for the SDSS SEGUE spectra and those of LAMOST Stellar Parameter Pipeline at PKU (LSP3) for the LAMOST spectra (Xiang et al. 2015a; Ren et al. 2016). Those large errors induce substantial uncertainties in other inferred parameters, such as stellar distance and age. The errors could also cause problems for classifying stars. For example, given the relatively narrow $(\sim 0.5 \mathrm{dex})$ range of values of $\log g$ of mainsequence turn-off/subgiant stars, samples of such stars selected based on stellar atmospheric parameters yielded by the aforementioned pipelines may suffer from significant contaminations from the numerous long-lived main-sequence dwarfs Xiang et al. 2015b). Similarly, for the evolved stars, as a result of the uncertainties in the derived parameters, stars of the red giant branch (RGB), the red clump (RC) and of the asymptotic giant branch (AGB) are mixed together in the $T_{\text {eff }}-\log g$ diagram and difficult to disentangled from each other (Huang et al. 2015b). Finally, little efforts has been made hitherto to estimate stellar luminosity directly from the stellar spectra, or to estimate the individual elemental abundances from low-resolution spectra.

One of the error sources for stellar atmospheric parameter determination comes from uncertainties in the template/training sets of spectra adopted. Both synthetic and empirical spectra have been widely used as the spectral templates or training sets. For synthetic templates, the fidelity of a spectrum for a given set of atmospheric parameters $\left(T_{\text {eff }}, \log g,[\mathrm{Fe} / \mathrm{H}],[\alpha / \mathrm{Fe}]\right)$ is limited by our knowledge of the often complicated stellar astrophysics and atomic/molecular opacities. A comprehensive comparison with the observed spectra of stars with accurate, independent determinations of atmospheric parameters over wide ranges of all atmospheric parameters is desirable to validate the synthetic spectra. For the empirical templates, on the other hand, both the spectra themselves and the atmospheric parameters associated with the stars, the latter often determined with a variety of techniques, have uncertainties, which eventually propagate into the parameters estimated for target stars. To obtain robust atmospheric parameters for large samples of stars targeted by large spectroscopic surveys, it seems logical to use a subset of the survey spectra whose atmospheric parameters have been previously accurately determined by other means as the template or training set to obtain parameter estimates for the remaining spectra, considering that in this case, both the template (training) set of spectra and target spectra are obtained with the same instrument and thus likely have the same error patterns. However, to obtain accurate stellar parameters by independent means for a substantial subset of spectra covering wide ranges of parameters is an extremely time-consuming and challenging task yet to be accomplished for the individual completed/on-going surveys.

For the LAMOST spectroscopic surveys, although a comprehensive subset of stellar spectra with accurately known atmospheric parameters is still absent, there are, however, thousands of stars targeted by LAMOST that have either accurate log $g$ measurements from asteroseismic analysis of the Kepler data (Huber et al. 2014), or precise measurements of metallicity [M/H], $\alpha$-element to metal abundance ratio $[\alpha / \mathrm{M}]$ and individual elemental abundances [X/H] for elements C, N, O, Na, Mg, Al, Si, S, K, Ca, Ti, V, Mn, $\mathrm{Fe}$ and $\mathrm{Ni}$ determined from the APOGEE high resolution spectra (Holtzman et al. 2015). There are also thousands of stars that have accurate Hipparcos parallax measurements (Perryman et al. 1997), thus their accurate luminosities can be derived. Those several sets of stars are therefore useful as training sets for the estimation of either stellar atmospheric parameters, luminosities or individual elemental abundances from the LAMOST spectra. Asteroseismic $\log g$ estimates inferred from the Kepler data can be accurate to 0.03 dex (Hekker et al. 2013; Huber et al.2014), much better than achievable even with high-resolution spectroscopy ( $\sim 0.1 \mathrm{dex})$, though $\log g$ values estimated from spectroscopy do not necessarily match fully with the asteroseismic results. The Hipparcos parallax measurements for stars in the solar neighborhood are accurate to a few per cent, corresponding to an accuracy of absolute magnitude better than about $0.2 \mathrm{mag}$. The APOGEE spectra have a resolution about 22500 (Majewski et al. 2010), much higher than that of the LAMOST spectra $(\sim 1800)$. Estimates of $[\mathrm{M} / \mathrm{H}],[\alpha / \mathrm{M}]$ and individual elemental abundances yielded by APOGEE spectra should be accurate/precise enough to be used for the LAMOST stellar parameter estimation.

Another important source of error for stellar atmospheric parameter determination comes from the inadequacy of algorithms used. Properties of an observed stellar spectrum are governed by the combination of a number of atmospheric parameters $\left(T_{\text {eff }},[\mathrm{Fe} / \mathrm{H}]\right.$, $[\alpha / \mathrm{Fe}], \log g$, etc.), thus parameters estimated from the spectrum are often degenerated, especially for a low-resolution spectrum where spectral features are often blended. Compared to $T_{\text {eff }}$, some parameters, in particular $\log g$, and some elemental abundances, are less sensitive to the observed spectral features. Estimates of those parameters therefore often suffer from lower accuracies if one uses the same metric (e.g. $\chi^{2}$ ) in pixel space of spectral flux for the estimation. The situation can be improved if specific spectral features can be singled out and used to estimate those parameters. However, singling out specific features sensitive to the individual parameters is not straightforward, due to the degeneracy of parameters as well as spectral blending, especially under low-resolution. The 'specific features', if exist, are likely to be non-linearly correlated with the observed flux in pixel space such that a non-linear algorithms is needed to find them out.

In this paper, we explore a multivariate regression method based on kernel-based principal component analysis (KPCA) to estimate atmospheric parameters, absolute magnitudes, and individual elemental abundances from the LAMOST spectra, utilizing the aforementioned spectral training sets. KPCA is a non-linear method to extract features from high-dimension data sets. It was first proposed by Schölpokf. Smola \& Müller (1998), and validated by a series of work (e.g. Müller et al. 2001; Zhang et al. 2005). Compared with the traditional (linear) PCA, KPCA can extract nonlinear components, thus one expects that it may yield higher accuracy for the estimation of stellar atmospheric parameters. For the purpose, we have defined four sets of training spectra consisting of: the MILES spectral library template stars, the LAMOSTHipparcos common stars, the LAMOST-Kepler common stars and the LAMOST-APOGEE common stars. We present a detailed analysis of the precisions of parameters yielded by these training sets, and estimate the parameter uncertainties as a function of signal-tonoise ratio (SNR) and stellar atmospheric parameters. The newly developed method will be incorporated into the LAMOST Stellar Parameter Pipeline at Peking University (LSP3; Xiang et al. 2015a), and the resultant parameters for stars of the LAMOST 
Spectroscopic Survey of the Galactic Anticentre will be publicly available in the second release of value-added catalogues of LSSGAC (LSS-GAC DR2; Xiang et al. 2016, in preparation).

The paper is arranged as follows. In $\S 2$, we briefly introduce the LAMOST Galactic surveys, data processing and parameter determination. In $\S 3$, we introduce our new method to estimate stellar parameters. The training sets are defined in $\$ 4$. In $\$ 5$, we present results applying the method to spectra collected by the LSS-GAC, including a detailed error analysis. In $\S 6$, we discuss briefly the potential future improvements of the method. This is followed by conclusions in $\S 7$.

\section{THE LAMOST GALACTIC SURVEYS}

The Large Sky Area Multi-Object Fiber Spectroscopic Telescope (LAMOST, also named as the "Guo Shoujing Telescope"; Wang et al. 1996; Cui et al. 2012) is a Wang - Su type reflecting Schmidt telescope that has both a wide field $\left(5^{\circ}\right.$ in diameter) and a large effective aperture $(4-6 \mathrm{~m}$, depending on the pointing altitude and hour angle). It collects simultaneously 4000 fiber spectra at a resolving power $R \sim 1800$ (with slit masks of $2 / 3$ the fiber diameter of $3.3 \mathrm{arcsec}$ ) of wavelength range $3700-9000 \AA$.

The LAMOST Galactic spectroscopic surveys - LAMOST Experiment for Galactic Understanding and Exploration (LEGUE; Zhao et al. 2012; Deng et al. 2012; Liu, Zhao \& Hou 2015), consist of three main components, the spheroid (Deng et al. 2012), disk (Hou et al. 2013) and Anti-center (LSS-GAC; Liu et al. 2014; Yuan et al.2015) surveys. In addition to the main surveys, there are projects targeting specific sky areas, such as the LAMOST-Kepler fields. The surveys aim to collect up to ten million stellar spectra down to 17.8 magnitude in the SDSS $r$-band (18.5 mag for limited fields). By June 2015, more than 5 million spectra have been obtained with SNRs higher than 10, the minimum required for a successful exposure (Liu et al. 2014; Yuan et al. 2015; Luo et al. 2015).

The raw 2-dimension data are processed with the LAMOST 2D pipeline for spectral extraction, wavelength calibration, flat fielding, background subtraction and flux calibration to produce 1D spectra (Luo et al. 2015). Given that the Anti-center survey (LSSGAC) targets fields of low Galactic latitudes that suffer from high dust extinction, a specific flux calibration pipeline has been developed at Peking University for accurate spectral flux calibration (Xiang et al. 2015c).

Two pipelines have been developed to derive stellar parameters, including radial velocity $V_{\mathrm{r}}$, effective temperature $T_{\text {eff }}$, surface gravity $\log g$ and metallicity $[\mathrm{Fe} / \mathrm{H}]$ from LAMOST spectra. The official LAMOST Stellar Parameter Pipeline (LASP; Wu et al. 2011, 2014; Luo et al. 2015) estimates parameters with a $\chi^{2}$ minimization scheme developed based on the ULySS procedure (Koleva et al. 2009; Wu et al. 2011, 2014), utilizing the ELODIE spectral library (Prugniel et al. 2007) as the templates. The LAMOST Stellar Parameter Pipeline at Peking University (LSP3; Xiang et al. 2015a; Ren et al. 2016) determines atmospheric parameters by template matching with the MILES spectral library (Sánchez-Blázquez et al. 2006), utilizing both a $\chi^{2}$-based weighted-mean and a $\chi^{2}$-minimization algorithm. Stellar atmospheric parameters of the MILES template stars have been (re-) homogenized by Huang et al. (2016, in preparation; cf. §4.1). A major observational campaign is currently under way to expand the MILES library (Wang et al. 2016, in preparation), by observing additional template stars in order to improve the coverage and ho- mogeneity of distribution of template stars in parameter space, as well as to extend the wavelength coverage of template spectra to the far red $(\sim 9000 \AA)$. By fixing $T_{\text {eff }}, \log g$ and $[\mathrm{Fe} / \mathrm{H}]$ yielded by the $\chi^{2}$-based weighted-mean algorithm, LSP3 also determines $[\alpha / \mathrm{Fe}]$ by template matching with Kurucz synthetic spectral library (Li et al. 2016). Stellar parameters yielded by LASP are publicly available from the LAMOST official data release1 Luo et al. 2012, 2015). Currently, LSP3 is only applied to spectra collected by the LSS-GAC survey, and the resultant parameters, as well as additional parameters such as interstellar dust extinction and distance, estimated based on the LSP3 atmospheric parameters with various methods, are released as value-added catalogues $2^{2}$ of LSS-GAC (Yuan et al.2015). Apart from results from the above two stellar parameter pipelines, Liu et al. (2015) use a support vector regression (SVR) method to estimate $\log g$ from LAMOST spectra for giant stars, taking the LAMOST-Kepler stars with asteroseismic $\log g$ measurements as the training set. Ho et al. (2016) estimate stellar atmospheric parameters $\left(T_{\text {eff }}, \log g,[\mathrm{Fe} / \mathrm{H}]\right.$ and $\left.[\alpha / \mathrm{Fe}]\right)$ for giant stars in the LAMOST DR2 by tying the LAMOST spectra to the APOGEE DR12 estimates of stellar parameters with the Cannon (Ness et al. 2015).

\section{THE METHOD}

\subsection{The Kernel-based Principal Components Analysis}

A detailed introduction of the Kernel-based PCA can be found in Schölpokf, Smola \& Müller (1998) and Müller et al. (2001). Below we briefly summarize the algorithm for completeness. Let $\boldsymbol{x}_{k}$, $k=1, \ldots, M$, denote the spectra of $m$ stars, each contains $N$ wavelength pixels, $\boldsymbol{x}_{k} \in \mathrm{R}^{N}$. The spectra are normalized such that the sum of all pixel squared values of a given spectrum is unity. Note that the normalization is critical to generate realistic values of the kernel function. To extract data structures with KPCA, we map the spectra into feature space $F$ by a (nonlinear) function $\Phi\left(x_{k}\right)$. Then we have the covariance matrix in $F$,

$$
C=\frac{1}{M} \sum_{i=1}^{M} \Phi\left(\boldsymbol{x}_{i}\right) \Phi\left(\boldsymbol{x}_{i}\right)^{\mathrm{T}} .
$$

To calculate the principal components, we solve the Eigenvalue problem below to find Eigenvalue $\lambda>0$ and Eigenvector $\boldsymbol{V} \neq 0$ :

$$
\lambda \boldsymbol{V}=C \boldsymbol{V}=\frac{1}{M} \sum_{i=1}^{M}\left(\Phi\left(\boldsymbol{x}_{i}\right) \cdot \boldsymbol{V}\right) \Phi\left(\boldsymbol{x}_{i}\right) .
$$

All Eigenvectors with nonzero Eigenvalue can be written in the span of $\Phi\left(\boldsymbol{x}_{1}\right), \ldots, \Phi\left(\boldsymbol{x}_{M}\right)$ such that,

$$
\boldsymbol{V}=\sum_{j=1}^{M} \alpha_{j} \Phi\left(\boldsymbol{x}_{j}\right)
$$

Multiplying Eq. (2) by $\Phi\left(\boldsymbol{x}_{k}\right)$ from the left yields,

$$
\begin{aligned}
& \lambda \sum_{j=1}^{M} \alpha_{j}\left(\Phi\left(\boldsymbol{x}_{k}\right) \cdot \Phi\left(\boldsymbol{x}_{j}\right)\right)= \\
& \frac{1}{M} \sum_{j=1}^{M} \alpha_{j} \sum_{i=1}^{M}\left(\Phi\left(\boldsymbol{x}_{k}\right) \cdot \Phi\left(\boldsymbol{x}_{i}\right)\right)\left(\Phi\left(\boldsymbol{x}_{i}\right) \cdot \Phi\left(\boldsymbol{x}_{j}\right)\right) .
\end{aligned}
$$

\footnotetext{
1 http://dr1.lamost.org

2 http://lamost973.pku.edu.cn/site/data
} 
Defining an $M \times M$ matrix $K$,

$$
K_{i j}:=\left(\Phi\left(\boldsymbol{x}_{i}\right) \cdot \Phi\left(\boldsymbol{x}_{j}\right)\right),
$$

the Eigenvalue problem becomes,

$$
M \lambda \boldsymbol{\alpha}=K \boldsymbol{\alpha},
$$

where $\boldsymbol{\alpha}=\left(\alpha_{1}, \ldots, \alpha_{M}\right)$. The solutions of the Eq. (6) are normalized by imposing $\lambda_{k}\left(\boldsymbol{\alpha}^{k} \cdot \boldsymbol{\alpha}^{k}\right)=1$. The normalization yields $\left(\boldsymbol{V}^{k} \cdot \boldsymbol{V}^{k}\right)=1$ for all $k=p, \ldots, M$, where $p$ is the index corresponding to the first nonzero Eigenvalue $\lambda_{p}$. The data in $F$ are centered by substituting $K$ with,

$$
\hat{K}=K-1_{M} K-K 1_{M}+1_{M} K 1_{M},
$$

where $\left(1_{M}\right)_{i j}=1 / M$.

To extract the principal components, we calculate the projections of the spectra on Eigenvectors $\boldsymbol{V}^{k}$ in $F$. For a given test spectrum $\boldsymbol{x}_{\boldsymbol{t}}$, the corresponding nonlinear principal components are

$$
\left(\boldsymbol{V}^{k} \cdot \Phi\left(\boldsymbol{x}_{\boldsymbol{t}}\right)\right)=\sum_{j=1}^{M} \alpha_{j}^{k}\left(\Phi\left(\boldsymbol{x}_{j}\right) \cdot \Phi\left(\boldsymbol{x}_{\boldsymbol{t}}\right)\right) .
$$

Even in the most realistic cases, the nonlinear transformation $\Phi$ in general can not be expressed explicitly. Therefore, instead of calculating the products $(\Phi(\boldsymbol{x}) \cdot \Phi(\boldsymbol{y}))$ in Eq. (6) directly, we use a kernel representation of the form,

$$
k(\boldsymbol{x}, \boldsymbol{y})=(\Phi(\boldsymbol{x}) \cdot \Phi(\boldsymbol{y})) .
$$

Various forms of kernel function, such as polynomial, radial basis functions and sigmoidal, as well as other more complicated kernels, have been validated (Schölpokf, Smola \& Müller 1998; Müller et al. 2001). In the current work, we use the Gaussian radial basis functions,

$$
k(\boldsymbol{x}, \boldsymbol{y})=\exp \left(\frac{-\|\boldsymbol{x}-\boldsymbol{y}\|^{2}}{c}\right),
$$

where $\|\cdot\|$ represents the Euclidean norm, $\|\boldsymbol{x}-\boldsymbol{y}\| \equiv$ $\sqrt{(\boldsymbol{x}-\boldsymbol{y}) \cdot(\boldsymbol{x}-\boldsymbol{y})}$, and $c$ is the width of the kernel. Throughout this paper, we adopt $c=0.005$, a typical value of the squared Euclidean norm $\|\boldsymbol{x}-\boldsymbol{y}\|^{2}$ for the LAMOST spectra. In fact, we have examined different values of $c$ (e.g. 0.005, 0.05, 0.5, 1.0, 5.0) making use of both LAMOST-Kepler sample stars and member stars of open clusters, and found that 0.005 is an optimal one.

\subsection{The Regression}

To derive atmospheric parameters from the principal components, we construct a multiple-linear relation between the principal components $\boldsymbol{P}$ and the stellar atmospheric parameters for each parameter $\boldsymbol{y}$,

$$
\boldsymbol{y}=\sum_{i=1}^{N} c_{i} \boldsymbol{P}_{i}+c_{0},
$$

where $N$ is the adopted number of principal components, determined empirically with a brute-force search (cf. $\S 4), c_{0}$ is a constant, and $\boldsymbol{y}$ is any one of the parameters $T_{\text {eff }}, \log g,[\mathrm{Fe} / \mathrm{H}], \mathrm{M}_{V}$, $\mathrm{M}_{K_{\mathrm{s}}},[\mathrm{M} / \mathrm{H}],[\alpha / \mathrm{M}],[\alpha / \mathrm{Fe}],[\mathrm{C} / \mathrm{H}]$ and $[\mathrm{N} / \mathrm{H}]$. The coefficients $c_{i}, i=1, \ldots, N$, are determined by a least square multiple-linear fit to a training data set.

When estimating $\log g$ values for giant stars using the LAMOST-Kepler sample stars as the training set (cf. §4.3), $T_{\text {eff }}$ and $[\mathrm{Fe} / \mathrm{H}]$ from LSP3 are adopted as priors. This is carried out by taking $\log T_{\text {eff }}$ and $[\mathrm{Fe} / \mathrm{H}]$ as input pixel values of spectral flux. Similarly, when estimating $\log g$ values for dwarfs using the MILES library as the training set (cf. §4.1), as well as when estimating $[\mathrm{M} / \mathrm{H}],[\alpha / \mathrm{M}]$ and $[\alpha / \mathrm{Fe}]$ using the LAMOST-APOGEE stars as the training set (cf. §4.4), $T_{\text {eff }}$ yielded by LSP3 is adopted as a prior. For the estimation of individual elemental abundances $[\mathrm{Fe} / \mathrm{H}],[\mathrm{C} / \mathrm{H}]$ and $[\mathrm{N} / \mathrm{H}]$ with the LAMOST-APOGEE training set, the LSP3 $T_{\text {eff }}$ as well as the KPCA estimated $[\mathrm{M} / \mathrm{H}]$ are adopted as the priors. Note however that, the priors are found to have only minor effects on the parameter estimation. The method remains robust even if incorrect values of $T_{\text {eff }}$ and $[\mathrm{Fe} / \mathrm{H}]$ are provided. This is probably due to the fact that LAMOST spectra contain sufficient information (features) to yield correct $\log g$ values and elemental abundances without relying on those priors. This also implies that our method is robust enough and is largely free from degeneracy of various parameters. Since the priors are not very helpful to improve the parameter estimation, for the estimation of absolute magnitudes using the LAMOST-Hipparcos stars as the training set (cf. §4.2) as well as the estimation of $T_{\text {eff }}$ and $[\mathrm{Fe} / \mathrm{H}]$ using the MILES stars as the training set (cf. §4.1), no priors are used for simplicity.

\subsection{Preprocessing for the spectra}

Though the LAMOST spectra cover a wavelength range of 3700 - $9000 \AA$, we have opted to use the $3900-5500 \AA$ segment only for the stellar parameter determination with the following considerations. Firstly, for the majority of stars in our sample, except for those very red ones, spectral features are mostly found in the blue part of the spectra, while the red part of the spectra contains much less features. The red part spectra also suffer from serious background contamination, including sky emission lines and telluric bands. Secondly, due to the low instrument efficiency near the edges of wavelength coverage of the blue- and red-arm spectra, for a considerable fraction of stars, the blue- and red-arm spectra are not perfectly jointed together but show artificial discontinuities. This is particularly serious for very bright stars observed under grey and bright lunar conditions and for faint stars of low spectral SNRs. Finally, the wavelength coverage of MILES spectra reach only $7410 \AA$ in the red. Note that the very blue part $(3700$ - $3900 \AA$ ) of the spectra is discarded because of the low spectral SNRs in this spectral regime for the majority of stars.

Given the relatively large uncertainties of spectral flux calibration of LAMOST spectra and the unknown extinction values for most of the survey targets, especially those within the Galactic disc, it is essential to use continuum-normalized spectra to deliever reliable stellar parameters. A reasonable continuum normalization is found to be crucial for robust estimation of stellar parameters. Since the MILES spectra are generally better calibrated and have higher SNRs than most LAMOST spectra, pesudo-continua determined for the former are in general more accurate. We therefore adopt the pesudo-continua determined for MILES spectra as a starting point to derive pesudo-continua for LAMOST spectra. Specifically, the pesudo-continua of MILES spectra are first derived using a 5th-order polynomial for the wavelength range $3850-5800 \AA$. The pesudo-continuum of a LAMOST spectrum is then derived by scaling that of the best-matching MILES spectrum as yielded by LSP3 with a 5th-order polynomial. Here the 5th-order polynomial is introduced to account for any differences of pesudo-continua between the LAMOST spectrum and the best-matching MILES spectrum. Such differences can be intrinsic or arise from differences in the interstellar extinction or are simply artifacts induced by inadequacies in the data reduction (e.g. uncertainties of flux calibration).

It is found that some LAMOST spectra have bad pixels of un- 
realistic fluxes as a consequence of inadequacy in the data reduction (e.g. poor cosmic ray removal). The presence of those bad pixels could have dramatic adverse effects on parameter estimation (cf. §5.1). To avoid the problem, we calculate the differences between the normalized LAMOST and best-matching MILES spectra, and identify bad pixels with $10 \sigma$ deviations. The spectral fluxes of bad pixels are replaced by values of Bessel interpolation of nearby pixels.

\section{THE TRAINING DATA SETS}

We define four training data sets in this work: the MILES spectral library, the LAMOST-Hipparcos common stars, the LAMOSTKepler stars with asteroseismic measurements of $\log g$ and the LAMOST-APOGEE common stars. The LAMOST-Kepler stars are used to estimate $\log g$ only, and the LAMOST-APOGEE stars are used to estimate metallicity $[\mathrm{M} / \mathrm{H}], \alpha$-element to metal abundance ratio $[\alpha / \mathrm{M}], \alpha$-element to iron abundance ratio $[\alpha / \mathrm{Fe}]$ and elemental abundance $[\mathrm{Fe} / \mathrm{H}],[\mathrm{C} / \mathrm{H}]$ and $[\mathrm{N} / \mathrm{H}]$. Both the LAMOSTKepler and LAMOST-APOGEE data sets are used to estimate parameters for giant stars only given that they contain few dwarfs. The MILES spectral library is used to estimate $T_{\text {eff }}$ and $[\mathrm{Fe} / \mathrm{H}]$ for all stars, and $\log g$ for dwarf and subgiant stars. Note that $[\mathrm{Fe} / \mathrm{H}]$ deduced using the MILES spectra as the training set are also referred to metallicity throughout the paper. The LAMOST-Hipparcos common stars, combined with the MILES stars with accurate measurements of parallax, are used to estimate absolute magnitudes. A summary of the adopted training sets and their effective parameter ranges, as well as the number of principle components adopted for the regression, are presented in Table 1. Note that for giant stars, there are two estimates of $[\mathrm{Fe} / \mathrm{H}]$, which are estimated based on the MILES training set and the LAMOST-APOGEE training set, respectively.

\subsection{The MILES spectral library}

The MILES spectral library includes 985 stars covering parameter space $3000<T_{\text {eff }}<40000 \mathrm{~K}, 0<\log g<5$ dex and $-3.0<[\mathrm{Fe} / \mathrm{H}]<0.5$ dex. The wavelength coverage of the spectra is $3525-7410 \AA$, and the spectral resolution is about $2.5 \AA$ (Sánchez-Blázquez et al. 2006; Falcón-Barroso et al. 2011). The latter is close to that of the LAMOST spectra. The MILES spectra were taken with a long-slit spectrograph, and specific efforts were carried out for accurate flux calibration (Sánchez-Blázquez et al. 2006), yielding stellar spectral energy distributions (SEDs) more reliable than possible with high-resolution echelle spectroscopy. The parameters of the MILES stars are collected from the literatures, mostly determined with high-resolution spectroscopy. Cenarro et al. (2007) have carried out a homogenization of the collected parameters to correct for systematics amongst values from different sources. Nevertheless, the homogenization of Cenarro et al. (2007) was carried out for a limited range of temperature, $4000<T_{\text {eff }}<6300 \mathrm{~K}$, and parameter values used for the template stars were obtained in early time (e.g. before 1990), with relatively large random errors. To reduce both the systematic and random errors of parameters of the MILES stars, Huang et al. (2016, in preparation) have recompiled usable determinations of parameters of the stars by replacing old measurements with more recent ones available from the PASTEL catalog Soubiran et al. 2010). The compiled values of effective temperature are then determined using the metallicity-dependent colour- temperature relations deduced from more than two hundred nearby stars with direct effective temperature measurements Huang et al. 2015a). Note that Huang et al.'s relations are only available for stars of $T_{\text {eff }}<10000 \mathrm{~K}$ and $[\mathrm{Fe} / \mathrm{H}]>-1.0$ dex. For stars outside those parameter ranges, the compiled temperatures are adopted. Values of surface gravity are re-determined using Hipparcos parallax (Perryman et al. 1997; Anderson \& Francis 2012) and stellar isochrones from the Dartmouth Stellar Evolution Database (Dotter et al. 2008). Finally, values of metallicity are re-calibrated to the scale of Gaia-ESO survey (Jofré et al. 2015). Here, we adopt those re-calibrated/determined parameters. Note that for the majority of stars, the re-calibrated/determined parameters are consistent well with the values of Cenarro et al. (2007).

MILES stars of $T_{\text {eff }} \leqslant 12000 \mathrm{~K}$ are selected as our training set for the estimation of $T_{\text {eff }}$ and $[\mathrm{Fe} / \mathrm{H}]$ for LAMOST stars. Stars of $T_{\text {eff }}>12000 \mathrm{~K}$ were excluded from the training set because tests show that if they were included, residuals of the multi-linear regression for $\mathrm{F} / \mathrm{G} / \mathrm{K}$ stars became very large. Here we concentrate on $\mathrm{A} / \mathrm{F} / \mathrm{G} / \mathrm{K}$ stars, and leave the parameter determination for stars of earlier-types a future work. Tests also show that excluding giant stars $(\log g<3.0$ dex $)$ in the training set yields significantly better results for dwarf and subgiant stars than otherwise. We therefore use MILES stars with $\log g>3.0$ dex only in the training sample since we want to determine accurate $\log g$ for dwarf and subgiant stars.

To select an optimal value for the number of principal components $N_{\mathrm{PC}}$ used for the regression, we compare the original MILES parameters with values deduced from the KPCA using different values of $N_{\mathrm{PCs}}$. The results are plotted in Fig. 1 . The Figure also compares the deduced $\log g$ of the LAMOST-Kepler dwarf/subgiant stars with the asteroseismic measurements. The Figure shows that as $N_{\mathrm{PC}}$ increases, the agreement generally improves. As $N_{\mathrm{pc}}$ increases from 20 to 100 , the dispersion of residuals of $T_{\text {eff }}$ decreases from $211 \mathrm{~K}$ to $143 \mathrm{~K}$, that of $\log g$ decreases from 0.22 dex to $0.13 \mathrm{dex}$, and that of $[\mathrm{Fe} / \mathrm{H}]$ decreases from 0.18 to 0.13 dex. Note that here the dispersion is a resistant estimate of the standard deviation. The comparisons of KPCA $\log g$ values deduced from the LAMOST spectra of the LAMOST-Kepler stars with asteroseismic $\log g$ measurements show that the systematic difference reaches a minimum of $0.00 \mathrm{dex}$ at $N_{\mathrm{PC}}=100$, and the dispersion is also small ( $0.11 \mathrm{dex})$. Although not plotted here, we have also carried out similar tests using candidate member stars of open clusters M 67, that have a significant number of member candidates targeted by the LAMOST. It is found that at an $N_{\mathrm{PC}}$ value around 100, the resultant $T_{\text {eff }}-\log g$ diagrams of cluster member star candidates match well with the theoretical isochrones. Based on the above tests, we have adopted $N_{\mathrm{PC}}=100$ for the MILES training set.

Note that though Fig. 1 shows that $N_{\mathrm{PC}}=100$ is the best for $\log g$ estimation, it is not necessary the best choice for the estimation of $T_{\text {eff }}$ and $[\mathrm{Fe} / \mathrm{H}]$ because the training samples are different - all MILES stars are used to estimate the latters, while only those of $\log g>3.0$ are used to estimate $\log g$. Nevertheless, we have adopted $N_{\mathrm{PC}}=100$ for the estimation of $T_{\text {eff }}$ and $[\mathrm{Fe} / \mathrm{H}]$ considering that for this $N_{\mathrm{PC}}$ value, the regression residuals are already quite small and acceptable. The selection of an optimal number of $N_{\text {PC }}$ if an effort to find a balance: although a larger value of $N_{\mathrm{PC}}$ will generate smaller residuals of the regression relations, an excessively large $N_{\mathrm{PC}}$ will also induce some undesired features caused by imperfections of the spectra. The latter could be potentially serious given that the training spectra (MILES) and the target spectra (LAMOST) are obtained with different instruments. In addition, 
Table 1. Adopted training sets, number of PCs and effective parameter ranges.

\begin{tabular}{llll}
\hline Training set & $N_{P C}$ & Parameters & Effective parameter range \\
\hline MILES & 100 & $T_{\text {eff }},[\mathrm{Fe} / \mathrm{H}]$ & $3000<T_{\text {eff }}^{\mathrm{LSP} 3}<12000 \mathrm{~K}$ \\
& & $\log g$ & $3000<T_{\text {eff }}^{\mathrm{LSP}}<12000 \mathrm{~K} \& \log g_{\mathrm{LSP} 3}>3.0$ \\
LAMOST-Hipparcos stars & 100 & $\mathrm{M}_{V}, \mathrm{M}_{K_{\mathrm{S}}}$ & $3000<T_{\text {eff }}^{\mathrm{LSP}}<12000 \mathrm{~K}$ \\
LAMOST-Kepler stars & 90 & $\log g$ & $T_{\text {eff }}^{\mathrm{LSP} 3}<5500 \mathrm{~K} \& \log g_{\mathrm{LSP} 3}<3.8$ \\
LAMOST-APOGEE stars & 90 & {$[\mathrm{M} / \mathrm{H}],[\mathrm{Fe} / \mathrm{H}],[\alpha / \mathrm{M}],[\alpha / \mathrm{Fe}],[\mathrm{C} / \mathrm{H}],[\mathrm{N} / \mathrm{H}]$} & $T_{\mathrm{eff}}^{\mathrm{LSP} 3}<5500 \mathrm{~K} \& \log g_{\mathrm{LSP} 3}<3.8$ \\
\hline
\end{tabular}

it is found that the optimal value of $N_{\mathrm{PC}}$ varies with the adopted value of kernel width $c$. In this paper, we have adopted a fixed value of $c(0.005)$. However, it is found that if we adopt a $c$ value of 0.5 , the optimal value of $N_{\mathrm{PC}}$ becomes $\sim 30$.

Fig. 2 plots the variations of regression residuals of $\log g$ estimates as functions of $T_{\text {eff }}$ and $[\mathrm{Fe} / \mathrm{H}]$. The left panel shows the residuals of $\log g$ of stars in different $[\mathrm{Fe} / \mathrm{H}]$ bins as a function of $T_{\text {eff }}$. It shows that the residuals of hot stars $\left(T_{\text {eff }}>7000 \mathrm{~K}\right)$ as well as metal-poor stars have larger dispersions than cooler, metal-rich stars. The dispersion is a measure of the precision of $\log g$ measurements of the MILES stars. The right panel shows the distribution of dispersions in the $T_{\text {eff }}-[\mathrm{Fe} / \mathrm{H}]$ plane. To calculate the dispersions, we first create a dense grid of $T_{\text {eff }}$ and $[\mathrm{Fe} / \mathrm{H}]$, with a step of $100 \mathrm{~K}$ and 0.1 dex in $T_{\text {eff }}$ and $[\mathrm{Fe} / \mathrm{H}]$, respectively. At each grid point, we search for the nearby training stars located within a box of size $100 \mathrm{~K}$ by $0.1 \mathrm{dex}$. If the number of stars within the box is smaller than 20 , we increase the box size by $50 \mathrm{~K}$ in $T_{\text {eff }}$ and $0.05 \mathrm{dex}$ in $[\mathrm{Fe} / \mathrm{H}]$ until the box size reaches an upper limit of $500 \mathrm{~K}$ by $0.5 \mathrm{dex}$. Finally, if the number of stars in the box is larger than 5, then the mean and standard deviation are calculated in a resistant way. Note that values of the dispersions shown in the Figure are square root of the mean of squares, i.e. the square of the mean and the square of the standard deviation. Here the square root of the mean of squares is preferred rather than the standard deviation because for some places of the parameter space (e.g. metal-poor side) or for bins where the numbers of stars are small, even though the computed standard deviation is small, the mean residual can be large. The Figure shows that the dispersions vary significantly over the $T_{\text {eff }}$ - $[\mathrm{Fe} / \mathrm{H}]$ plane. For $T_{\text {eff }}<7000 \mathrm{~K}$ and $[\mathrm{Fe} / \mathrm{H}]>-1.0 \mathrm{dex}$, the dispersions can be as small as $\sim 0.1 \mathrm{dex}$, indicating a high precision of $\log g$ determination for the F/G/K-type metal-rich stars. For metal-poor $([\mathrm{Fe} / \mathrm{H}]<-1.0 \mathrm{dex})$ or relatively hot $\left(T_{\text {eff }}>7000 \mathrm{~K}\right)$ stars, the dispersions are significantly larger. Such an error pattern is consistent with the results shown in Fig. 1, where the dispersions of the overall residuals of MILES stars could be significantly larger than those deduced for the LAMOST-Kepler stars, since the latter consist entirely of F/G stars with $5000<T_{\text {eff }}<7000 \mathrm{~K}$.

\subsection{The LAMOST-Hipparcos stars}

The LAMOST has collected spectra of many very bright stars utilizing moon nights. A cross-identification of the LAMOST stars observed before Oct. 2015 with the Hipparcos catalog (Perryman et al. 1997) yields more than 7000 common stars. A considerable fraction of these Hipparcos stars have accurate measurements of parallax and magnitudes, such that their luminosity (absolute magnitude) can be derived with good accuracy. We take those stars as a training set to estimate directly stellar absolute magnitudes from the LAMOST spectra. To construct this data set, we require that the training stars have uncertainties of the absolute magnitudes, which are propogated from the parallax errors, smaller

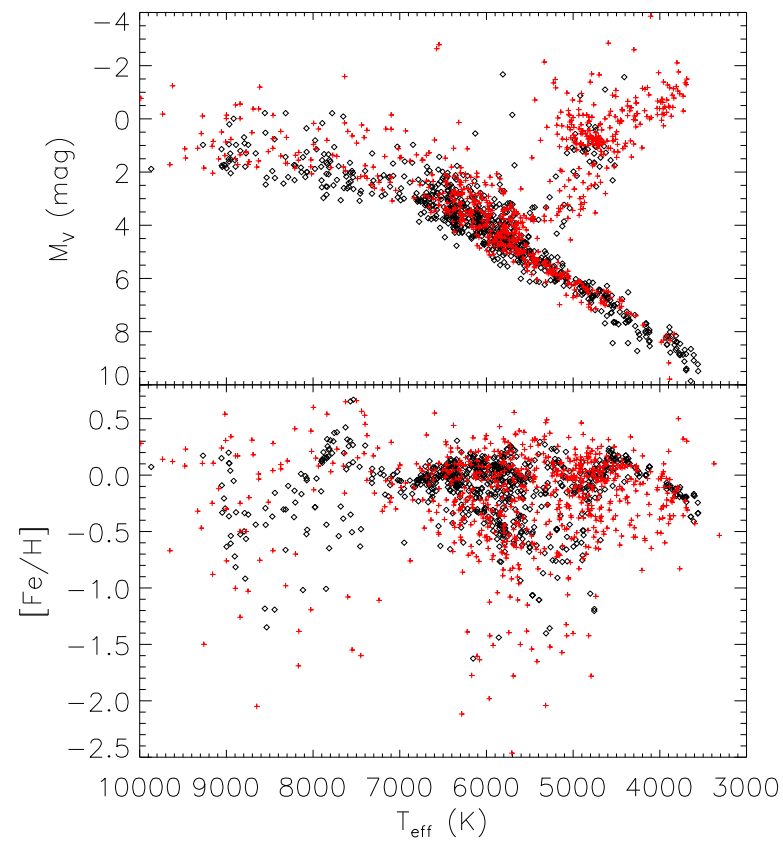

Figure 3. Distribution of the luminosity training stars in the $T_{\text {eff }}-\mathrm{M}_{V}$ and $T_{\text {eff }}-[\mathrm{Fe} / \mathrm{H}]$ plane. The black diamonds are the LAMOST-Hipparcos stars, while the red plus are the MILES stars. $T_{\text {eff }}$ and $[\mathrm{Fe} / \mathrm{H}]$ of the LAMOSTHipparcos stars are derived with the LSP3.

than $0.3 \mathrm{mag}$, and we also require the training star have a LAMOST SNR higher than 50. These criteria lead to 810 stars left in our training data set.

Though most of the training stars are local enough so that the inter-stellar extinction are negligible, a few of them are found to suffer considerable extinction $\left(E_{B-V} \sim 0.1 \mathrm{mag}\right)$, and need to be corrected for. To derive extinction for those training stars, we deduce the intrinsic colour $\left(V-K_{\mathrm{s}}\right)_{0}$ utilizing stellar atmospheric parameters derived from the LAMOST spectra with the LSP3 and the metallicity-dependent colour-temperature relation of Huang et al. (2015a). The distribution of the deduced $E_{B-V}$ has an overall peak at $0.01 \mathrm{mag}$, with a dispersion of $0.03 \mathrm{mag}$, as well as a small tail which corresponds to stars with significant extinction. We therefore correct for extinction for 161 stars both of $E_{B-V}>0.03$ mag and with distance larger than $50 \mathrm{pc}$.

Considering the number of stars in the training set is small, and more important, there are rare metal-poor $([\mathrm{Fe} / \mathrm{H}]<-1.0)$ stars, we choose to add 742 MILES stars in the training set. Those MILES stars have also accurate parallax from the Hipparcos cata$\log$, which result in an uncertainty in absolute magnitude smaller than 0.3 mag. As a result, there are 1552 training stars in total for the estimation of stellar luminosity. The distribution of those train- 

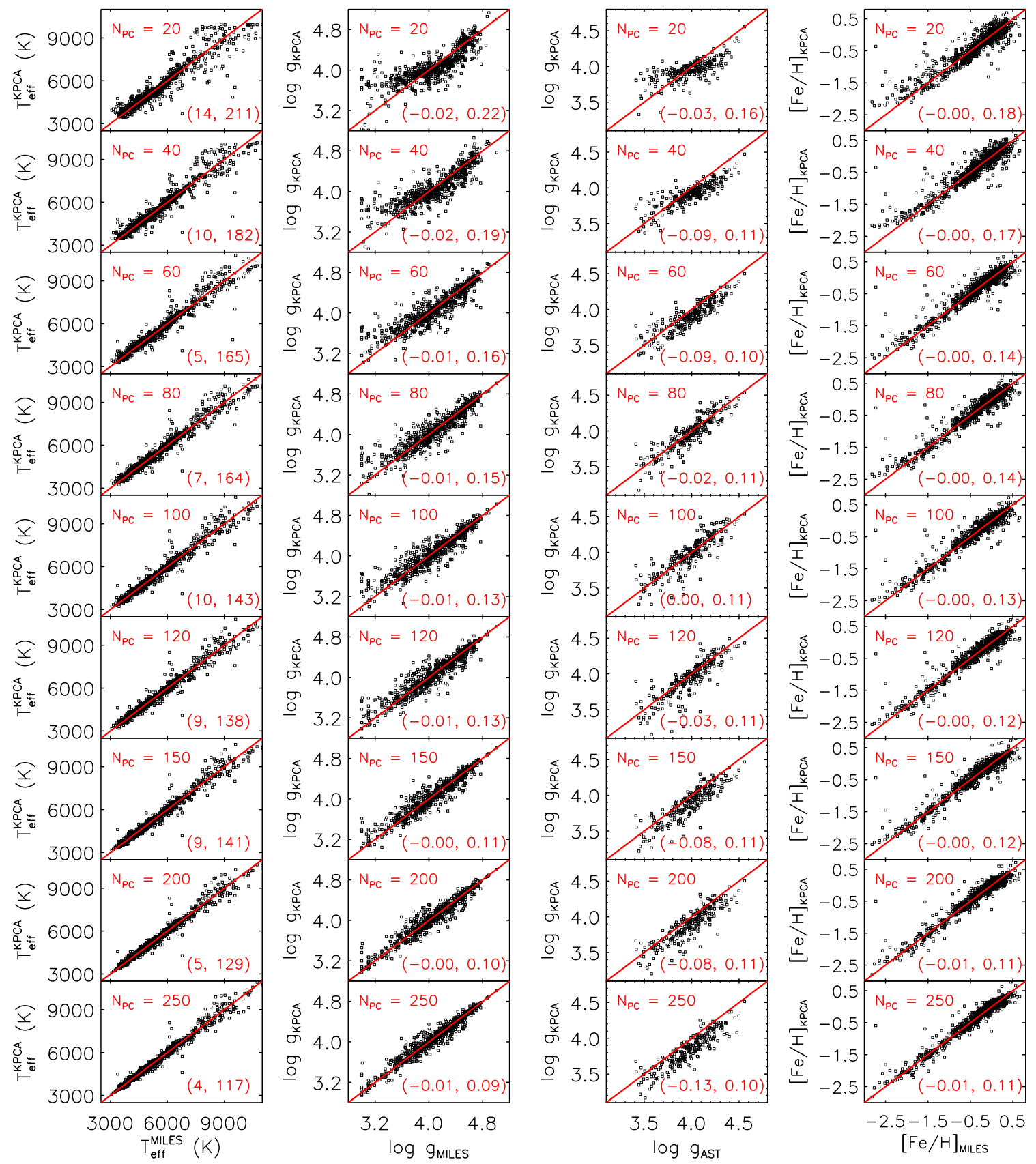

Figure 1. Comparison of KPCA atmospheric parameters with the MILES values (Columns 1,2 and 4 for $T_{\text {eff }}$, $\log g$ and [Fe/H], respectively.) for the MILES training stars. The KPCA $\log g$ estimates are compared to the asteroseismic measurements $\log g_{\text {AST }}$ in Column 3 for the LAMOST-Kepler stars of $\log g_{\mathrm{AST}}>3.4 \mathrm{dex}$. For each column, different panels show results for different number of principal components adopted. The number of principal components, as well as a resistant estimates of the mean and standard deviation of the parameter differences, are marked in the plots.

ing stars in the $T_{\text {eff }}-\mathrm{M}_{V}, T_{\text {eff }}-[\mathrm{Fe} / \mathrm{H}]$ plane are shown in Fig. 3, with the LAMOST-Hipparcos stars and the MILES stars shown in different symbols.

To select the optimal number of PC used for the regression, we have examined results deduced assuming different numbers of PC from 20 to 500 for a test data set, which contains both LAMOSTHipparcos stars of SNR smaller than 50 and counterpart stars of duplicate observations of the training stars, as well as open cluster member star candidates. Finally, we adopt 100 PCs for the multiple linear regression. Figure 4 shows the residuals of the regression for the Johnson $V$ and the 2MASS $K_{\mathrm{s}}$-bands absolute magnitudes as functions of effective temperature and metallicity. Here for the LAMOST-Hipparcos training stars, the temperature and metallicity are values yielded by LSP3. The Figure shows that though the dispersion of the residuals for stars of higher temperature or lower metallicity are slightly larger, there is no visible bias of the mean 

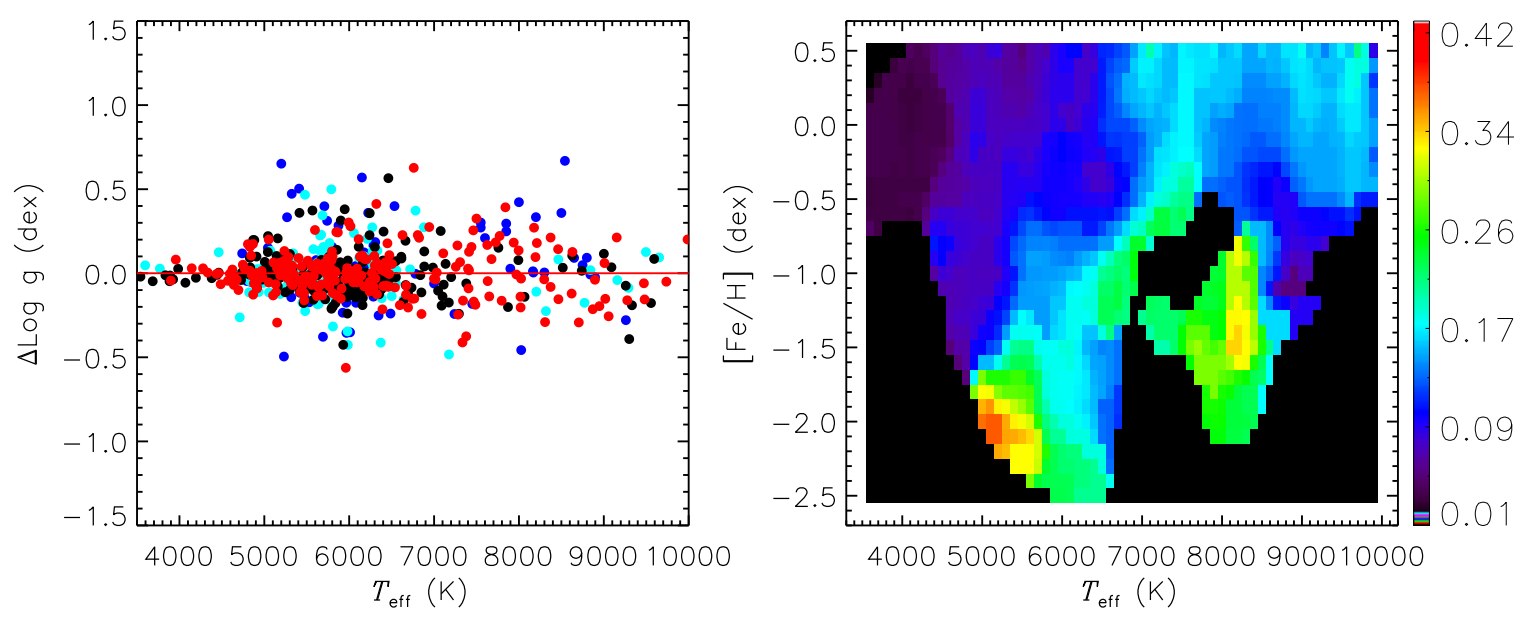

Figure 2. Residuals of regression of $\log g$ estimation utilizing the MILES library as the training data set. Left: Residuals of log $g$ of MILES stars in different metallicity bins (red: $[\mathrm{Fe} / \mathrm{H}]>0$; black: $-0.5<[\mathrm{Fe} / \mathrm{H}]<0$; cyan: $-1.0<[\mathrm{Fe} / \mathrm{H}]<-0.5$; blue: $[\mathrm{Fe} / \mathrm{H}]<-1.0$ dex); Right: Distribution of the dispersions of $\log g$ residuals in the $T_{\text {eff }}-[\mathrm{Fe} / \mathrm{H}]$ plane.

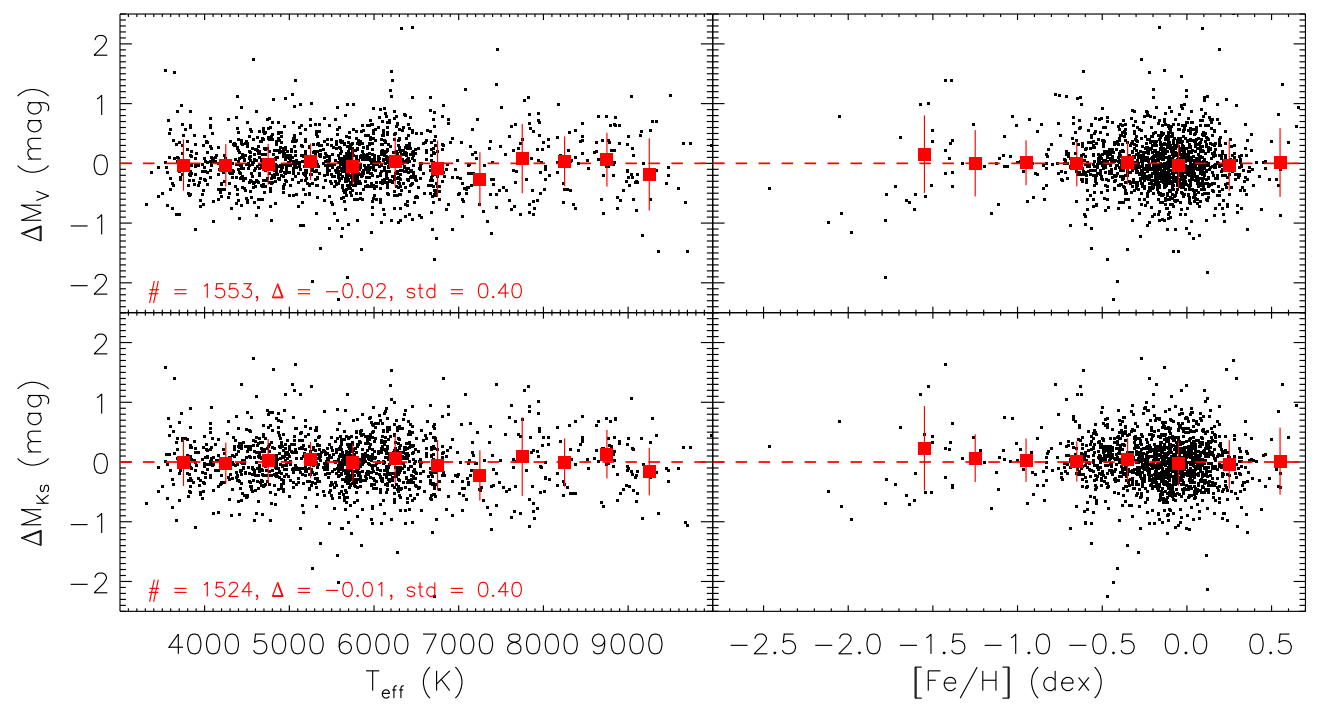

Figure 4. Residuals of absolute magnitudes for the LAMOST-Hipparcos training stars as a function of effective temperature and metallicity. The upper panels are results for the Johnson $V$-band magnitude, while the lower panels are results for the 2MASS $K_{\mathrm{S}}$-band magnitude. The number of the training stars, as well as the mean and dispersion of the distribution of the residuals estimated with a Gaussian fit, are marked in the plot.

residuals respect to the temperature and metallicity. Values of the mean and standard deviation of the overall residuals are respectively -0.02 and 0.40 mag for the $V$-band absolute magnitude, and -0.01 and $0.40 \mathrm{mag}$ for the $K_{\mathrm{s}}$-band absolute magnitude.

\subsection{The LAMOST-Kepler stars}

The LAMOST-Kepler project targets stars in the Kepler fields with the LAMOST, and more than 100000 spectra have been collected by September, 2014 (De Cat et al. 2015). We have processed the raw LAMOST-Kepler data collected since the beginning of the LAMOST pilot survey initiated in October, 2011 until June 2014, using the LAMOST 2D pipeline (Luo et al. 2015) and the flux calibration pipeline developed for the LSS-GAC survey (Xiang et al. 2015c). The data set includes about 53000 spectra of
SNR higher than 10 per pixel at $4650 \AA$. The stellar atmospheric parameters are derived from the spectra with LSP3. Amongst them, 3954 unique stars are found to have asteroseismic measurements of $\log g$ from Huber et al. (2014). Most of them (3709) are giant stars of $\log g_{\mathrm{AST}}<3.4$ dex. Only 245 stars have $\log g_{\mathrm{AST}}$ values larger than 3.4 dex.

The common stars are divided into two samples, a training sample and a test sample. The training sample is used to generate regression relations between the principal components and the stellar parameters, while the test sample is used to evaluate the generated relations. To select stars of the training sample, we first discard stars with a spectral SNR lower than 50, then divide the remaining stars into small cells in the $T_{\text {eff }}-\log g-[\mathrm{Fe} / \mathrm{H}]$ space. Here $T_{\text {eff }}$ and $[\mathrm{Fe} / \mathrm{H}]$ refer to values derived from the LAMOST spectra with LSP3, and $\log g$ refers to the asteroseimic values. The training stars 

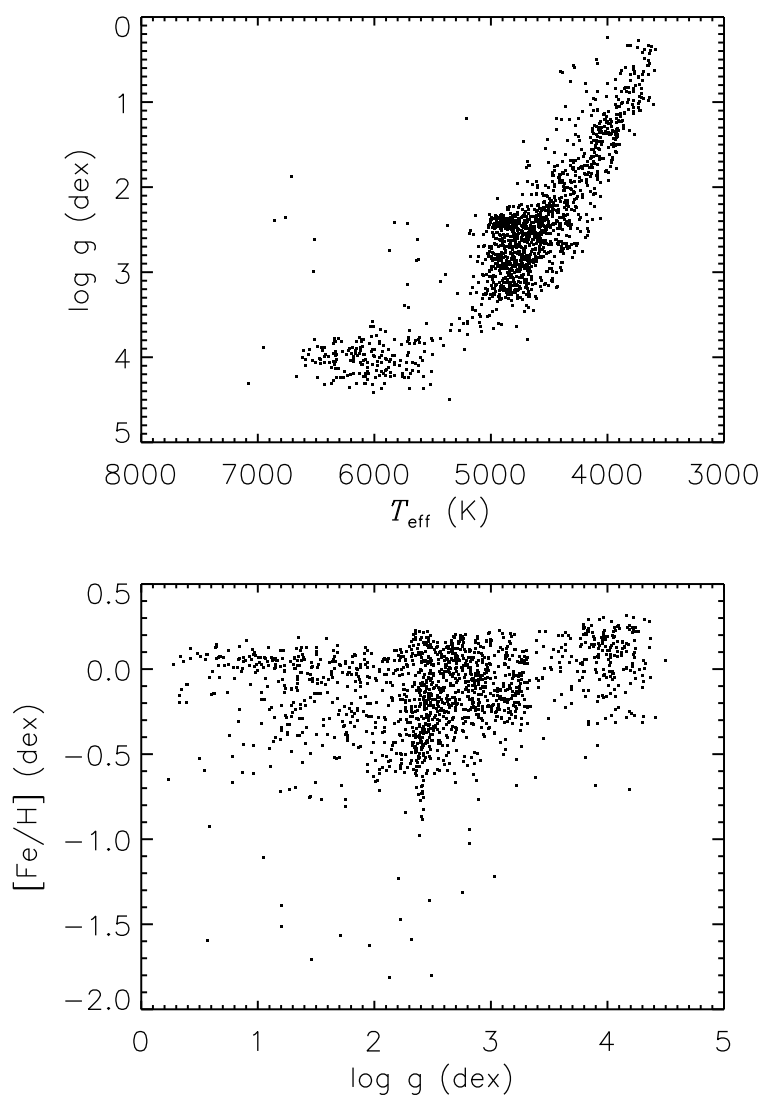

Figure 5. Distribution of the LAMOST-Kepler training stars in the $T_{\text {eff }}$ $-\log g$ and $\log g-[\mathrm{Fe} / \mathrm{H}]$ planes. Values of $T_{\text {eff }}$ and $[\mathrm{Fe} / \mathrm{H}]$ are those yielded by LSP3, while those of $\log g$ are asteroseismic measurements from Huber et al. 2014).

are then randomly selected from the individual cells. The selection is to ensure that the training stars are distributed as widely and homogeneously as possible in the parameter space. Half of the stars with a spectral SNR higher than 50 are selected as the training stars. The other half, together with those with a spectral SNR lower than 50, are adopted as the test sample. Although we intend to use this training set to estimate $\log g$ for giant stars only (cf. $§ 1$ ), it is found that keeping a sufficient number of dwarf stars in the training sample is necessary to avoid systematic errors of $\log g$ deduced for stars of $\log g>3.0$ dex. Huang et al. (2015b) shows that there is an artificial systematic trend in the KPCA estimates of $\log g$ for stars of a $\log g$ value larger than 3.0 dex. The artifacts disappear if dwarf stars are also included in the training set. Considering that the number of dwarf stars are very small in our sample, we include all stars of $\log g>3.4$ dex in our training set. In total, the training set contains 1520 stars. Fig. 5 shows the distribution of the training sample in the $T_{\text {eff }}-\log g$ and $\log g-[\mathrm{Fe} / \mathrm{H}]$ planes. The Figure shows that most of the stars have a metallicity $[\mathrm{Fe} / \mathrm{H}]>-1.0 \mathrm{dex}$, suggesting a disk sample.

Since this LAMOST-Kepler training set is only used for log $g$ estimation, the values of $T_{\text {eff }}$ and $[\mathrm{Fe} / \mathrm{H}]$ of stars in the sample given by the LSP3 are adopted as prior to increase the robustness of results. To choose an optimal number of PCs for the multi-linear regression, we carry out tests similar to those done for the MILES training set. We have also applied different numbers of PCs to the test sample, and examine the robustness of the residuals. Finally, we adopt a number of 90 PCs and a kernel-width of 0.005 . Note that the optimal number of PCs changes with the adopted kernelwidth. Generally, it is found that for a larger kernel-width, a smaller number of PCs is required. For instance, if one adopts a the kernelwidth of 0.5 , a number of about 30 PCs seems to yield the optimal results. Fig. 6 shows the regression residuals of $\log g$ of the training stars for a kernel-width $c=0.005$ and a number of PCs $N_{p c}=90$. The mean of the overall residuals is $-0.01 \mathrm{dex}$, with a dispersion of 0.11 dex. The residuals show no significant trends with $T_{\text {eff }}, \log g$ and $[\mathrm{Fe} / \mathrm{H}]$ for stars of $\log g<4.0 \mathrm{dex}$ and $[\mathrm{Fe} / \mathrm{H}]>-1.0$ dex. Given that there are few of very metal-poor training stars, results for more metal-poor stars are probably less accurate. Values of $\log g$ for stars of $\log g>4.0$ dex may have been underestimated. Since we focus on giant stars with this training set, we have ignored this potential systematics.

\subsection{The LAMOST - APOGEE common giant stars}

The SDSS-III/APOGEE collects $H$-band infrared spectra (1.51 $-1.70 \mu \mathrm{m}$ ) at a resolving power $R \sim 22500$ (Majewski et al. 2010). Stellar atmospheric parameters $\left(T_{\mathrm{eff}}, \log g,[\mathrm{M} / \mathrm{H}]\right)$, abundance ratios $[\mathrm{C} / \mathrm{M}],[\mathrm{N} / \mathrm{M}]$ and $[\alpha / \mathrm{M}]$, as well as elemental abundances $[\mathrm{X} / \mathrm{H}]$ for 15 individual elements are deduced from the spectra with the Apogee Stellar Parameter and Chemical Abundance Pipeline (ASPCAP) via template matching with a synthetic spectral library. For determinations of $T_{\text {eff }}, \log g,[\mathrm{M} / \mathrm{H}],[\mathrm{C} / \mathrm{M}]$, $[\mathrm{N} / \mathrm{M}]$ and $[\alpha / \mathrm{M}]$, the full APOGEE spectra are used for template matching, while for determinations of the individual elemental abundances, specific segments of spectra are selected for template matching (García Pérez et al. 2016; Holtzman et al. 2015). Systematic trends in the resultant metallicity $[\mathrm{M} / \mathrm{H}]$ and elemental abundances $[\mathrm{X} / \mathrm{H}]$ (except for carbon abundance $[\mathrm{C} / \mathrm{H}]$ and nitrogen abundance $[\mathrm{N} / \mathrm{H}])$ as a function of $T_{\text {eff }}$ are corrected for using member stars of open and globular clusters, yielding a final internal accuracy of $0.05-0.10 \mathrm{dex}$. For some elements, the internal accuracy of abundance is even better than $0.05 \mathrm{dex}$ at high spectral SNR (Holtzman et al. 2015). Values of [M/H] have also been calibrated externally to account for the observed systematic trends with the metallicities $[\mathrm{Fe} / \mathrm{H}]$ of star clusters. Though no external calibration of individual elemental abundances against independent data set have been carried out, the overall trend of $[\alpha / \mathrm{M}]$ with $[\mathrm{M} / \mathrm{H}]$, as well as the overall trends of individual elemental abundances with $[\mathrm{Fe} / \mathrm{H}]$, seem to match well those deduced from high resolution spectroscopy of stars in the solar neighborhood (e.g. Bensby, Feltzing \& Lundström 2003). The SDSS DR12 catalog includes 163278 APOGEE stars in total, and 102178 of them are giant stars with calibrated stellar parameters (Holtzman et al. 2015).

A cross-identification of the LSS-GAC catalog with APOGEE giant stars of $3500<T_{\text {eff }}<5300 \mathrm{~K}$ and $\log g<3.8$ dex yields 8400 common objects that have a LAMOST spectral SNR higher than 10 . Here $T_{\text {eff }}$ and $\log g$ refer to the APOGEE values. To derive principal components accurately, we include only stars with a spectral SNR higher than 50 in the training set. This yields a total of 3533 stars in the set. We divide the stars into small cells in the $[\mathrm{M} / \mathrm{H}]-[\alpha / \mathrm{M}]$ plane, and select half of them (1766) uniformly and randomly from the individual cells as our training sample. The other half, as well as common objects of a LAMOST spectral SNR lower than 50, are selected as the test set. Fig. 7 shows the distribution of the training stars in the $T_{\text {eff }}-\log g$ and the $[\mathrm{M} / \mathrm{H}]-[\alpha / \mathrm{M}]$ planes. With this training set, we estimate the metallicity $[\mathrm{M} / \mathrm{H}]$, $\alpha$-element to metallicity ratio $[\alpha / \mathrm{M}], \alpha$-element to iron abundance 


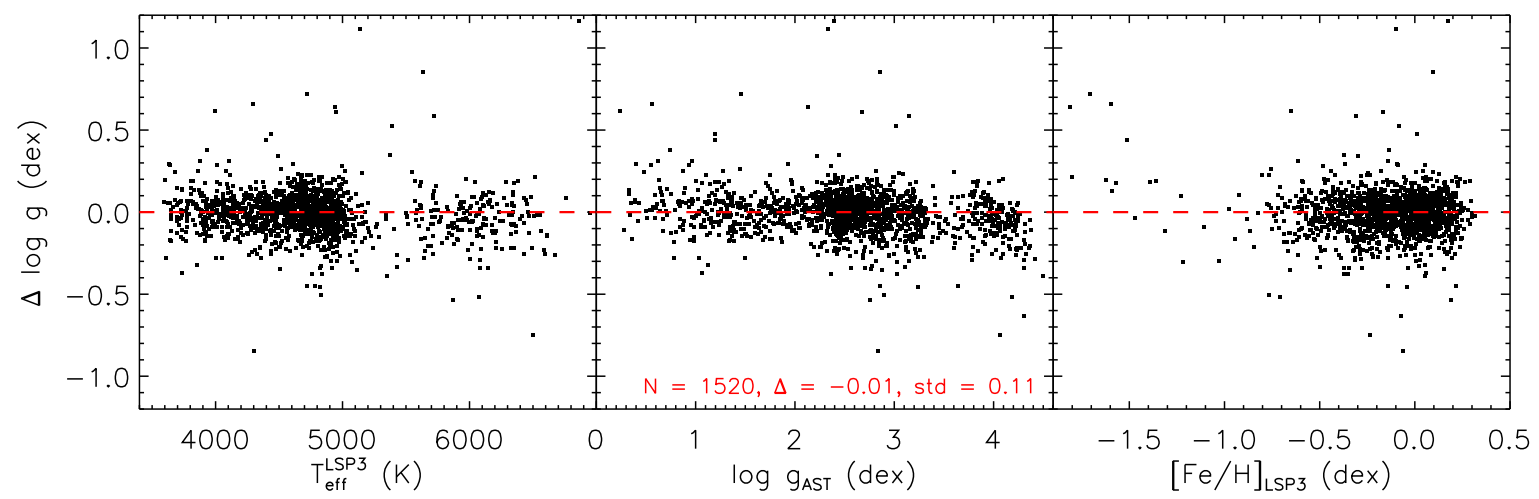

Figure 6. Residuals of regression of $\log g$ estimation utilizing the LAMOST-Kepler stars as the training data set. The residuals are shown as a function of stellar parameters. The number of training stars, as well as resistant estimates of the mean and standard deviation of the residuals are marked in the middle panel.
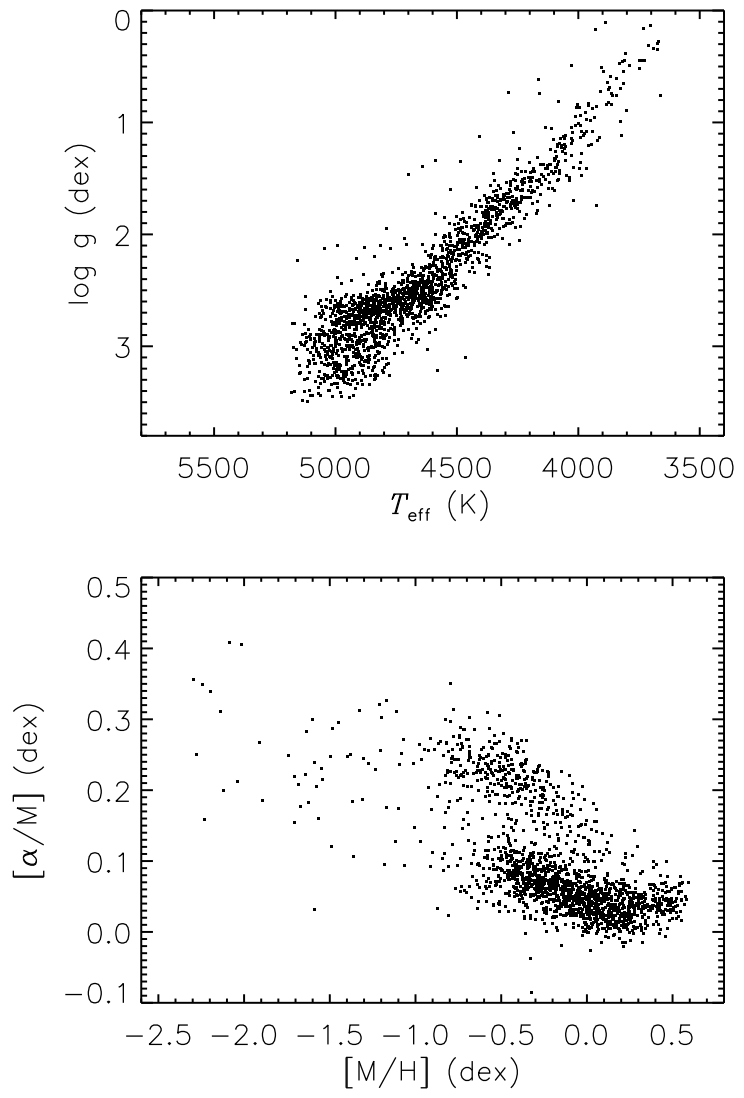

Figure 7. Distribution of the LAMOST-APOGEE training stars in the $T_{\text {eff }}$ $-\log g$ and $[\mathrm{M} / \mathrm{H}]-[\alpha / \mathrm{M}]$ planes. Values of $T_{\text {eff }}$ are those derived with LSP3, while those of $\log g,[\mathrm{M} / \mathrm{H}]$ and $[\alpha / \mathrm{M}]$ are from the APOGEE catalog Holtzman et al. 2015).

ratio $[\alpha / \mathrm{Fe}]$ and elemental abundances $[\mathrm{C} / \mathrm{H}],[\mathrm{N} / \mathrm{H}]$ and $[\mathrm{Fe} / \mathrm{H}]$. An investigation on deriving abundances of other individual elements from LAMOST spectra will be presented elsewhere. Here we also do not estimate effective temperature and surface gravity, because the current LSP3 can provide effective temperature with good accuracy, and we have adopted the LAMOST-Kepler stars with asteroseismic measurements of surface gravity as the training data set for the estimation of surface gravity for LAMOST giant stars.

Similar to the case of the LAMOST-Kepler training set, we adopt a number of 90 PCs for the multiple-linear regression. Fig. 8 plots a comparison of the regressed parameters with the APOGEE values for the training stars. The Figure shows that the APOGEE parameters are all well reproduced by the regression, and the standard deviations of the residuals are only 0.07 dex for $[\mathrm{M} / \mathrm{H}], 0.03 \mathrm{dex}$ for $[\alpha / \mathrm{M}], 0.04 \mathrm{dex}$ for $[\alpha / \mathrm{Fe}]$ and $0.04-0.08 \mathrm{dex}$ for $[\mathrm{Fe} / \mathrm{H}],[\mathrm{C} / \mathrm{H}]$ and $[\mathrm{N} / \mathrm{H}]$. The small standard deviations not only demonstrate the feasibility of our method, but also validate the precisions of the APOGEE parameters. Values of standard deviation for the metal-poor stars (e.g. $[\mathrm{M} / \mathrm{H}]<1.0 \mathrm{dex})$ are larger than those for the metal-rich stars, this is because the APOGEE parameters are less accurate for the metal-poor stars. Since there are few training stars of $[\mathrm{M} / \mathrm{H}]<-2.0 \mathrm{dex}$, and the parameters of those stars are also quite uncertain, our method probably systematically overestimates metallicity and elemental abundances for stars of metallicity below $-2.0 \mathrm{dex}$. Similarly, our method probably underestimates $[\alpha / \mathrm{M}]$ and $[\alpha / \mathrm{Fe}]$ for stars of $[\alpha / \mathrm{M}]>0.3 \mathrm{dex}$. Note that since abundances of the APOGEE measurements are not externally calibrated to standard absolute scales, systematic offsets or bias could be hided in the APOGEE abundances, which may also have been propagated into our results.

Although the standard deviations of regression residuals are small for all abundances, it not necessarily means that all the abundances are direct and reliable estimates, which reflect abundances of the individual elements. This is because our method is deducing abundance using all spectral features, not only those of the element concerned. As a consequence, it is possible that abundances of some elements are tied to those of other elements artificially, if the former contribute too weak features in the spectra. To examine such artifacts, we further compare abundances ratios $[\mathrm{X} / \mathrm{Y}]$ deduced from our abundance estimates with those of the APOGEE values for the test star sample. Here $\mathrm{X}$ (and Y) refers to any of the metal $\mathrm{M}$, alpha-elements $\alpha$, iron $\mathrm{Fe}$, carbon $\mathrm{C}$ and nitrogen $\mathrm{N}$, and the $\alpha$ abundance is transferred from $[\alpha / \mathrm{Fe}]$ and $[\mathrm{Fe} / \mathrm{H}]$. For the comparison, we select stars with a LAMOST spectral SNR higher than 50 and with a APOGEE spectral SNR higher than 60, and we further discard stars with uncertainties of the APOGEE abundances larger than 0.1 dex. The comparisons, as well as correlation coefficients between our abundance ratio estimates and those of the 

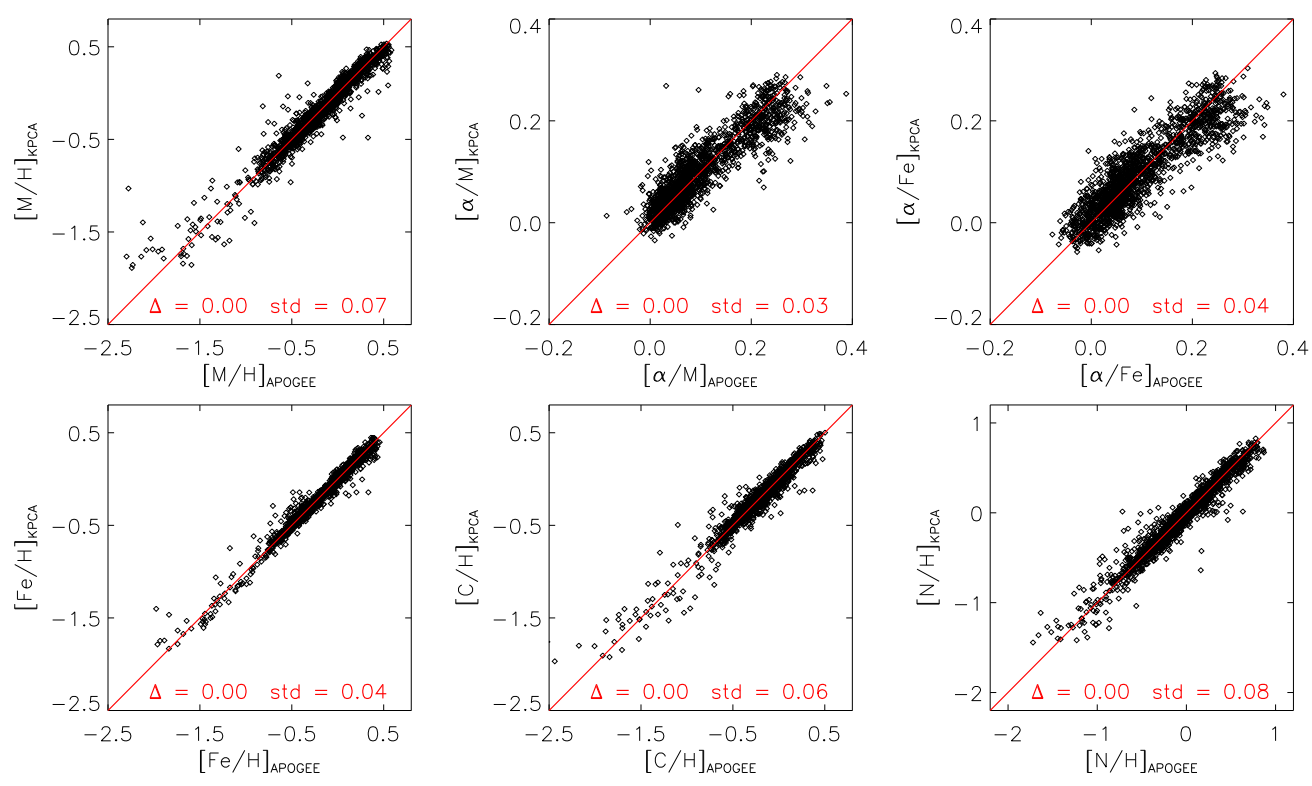

Figure 8. Comparison of abundances between the regressed values and the APOGEE measurements for the LAMOST-APOGEE training stars. Resistant estimates of the mean and standard deviation of the residuals are marked in the plots.

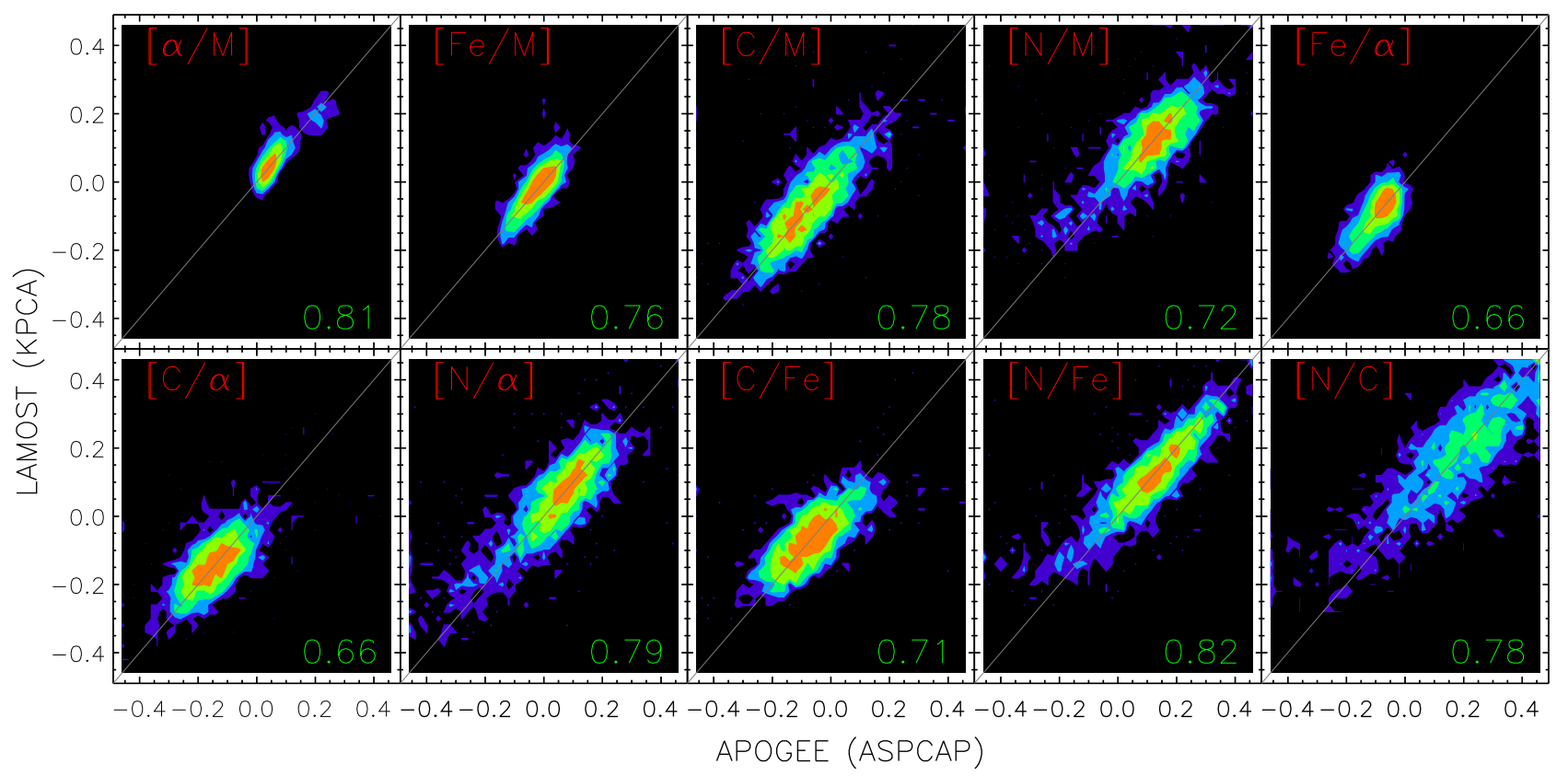

Figure 9. Comparison of elemental abundance ratios between the KPCA and APOGEE results for the LAMOST-APOGEE test star sample. Colours indicate stellar number density in logarithmic scale. The number in each plot labels linear correlation coefficient calculated with the individual stars.

APOGEE, are shown in Fig. 9. The Figure shows that for all abundance ratios, our results are in good agreement with the APOGEE values, indicating our abundances are reliable estimates. We also inspect the LAMOST spectra in given limited ranges of $T_{\text {eff }}, \log g$, and $[\mathrm{Fe} / \mathrm{H}]$, and find that strength of spectral features of $\mathrm{C}, \mathrm{N}$ and $\alpha$-elements (e.g. CN $\lambda 4215 \AA, C H \lambda 4314 \AA$, Ca I $\lambda 4226 \AA$ etc.) are correlated well with the estimated abundances.

\section{RESULTS}

By June, 2014, LSS-GAC has collected more than 1.8 million LSSGAC spectra with a spectral SNR higher than 10 for about 1.4 million unique stars. Fundamental stellar atmospheric parameters ( $\left.T_{\text {eff }}, \log g,[\mathrm{Fe} / \mathrm{H}]\right)$ have been deduced from these spectra with LSP3 (v1) via a template-matching technique utilizing the MILES spectral library. Here we derive stellar parameters from these spectra with the KPCA-based regression. Note that the LSS-GAC spec- 

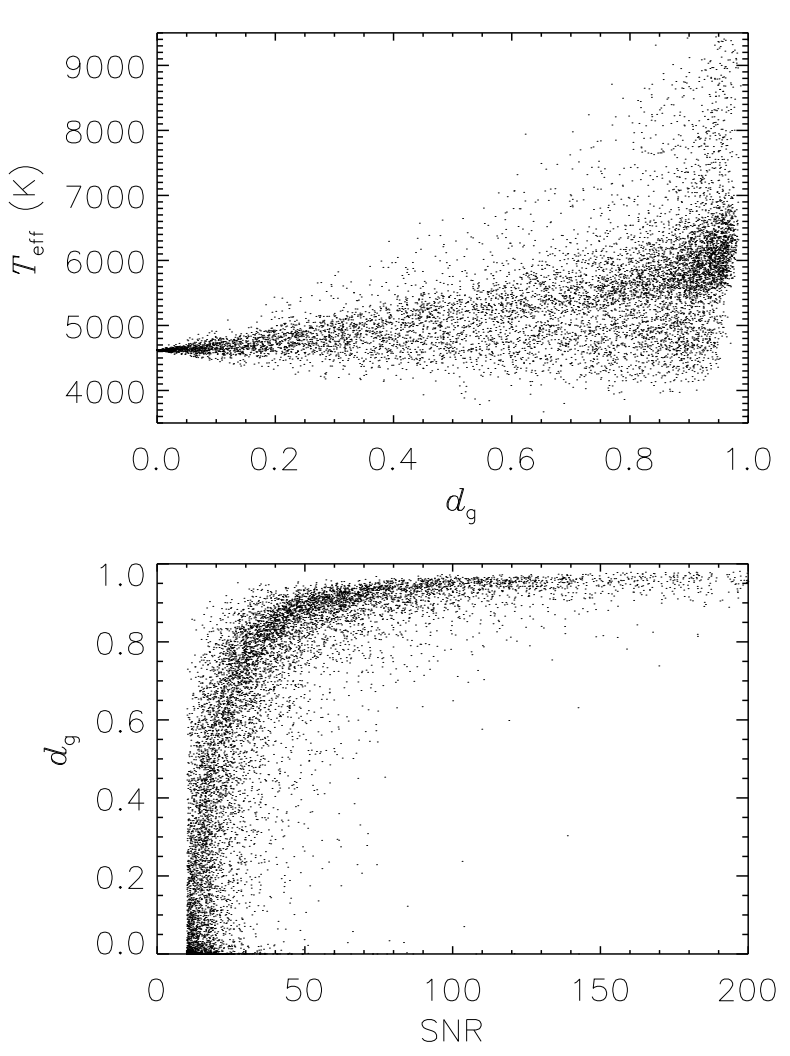

Figure 10. Upper panel: The estimated effective temperatures as a function of $d_{\mathrm{g}}$ (see the text $\S 5.1$ ). Lower panel: The $d_{\mathrm{g}}$ values as a function of the spectral signal-to-noise ratio. Only results of 10000 stars randomly selected from the whole sample are shown in the plots.

tra used in this work are processed at Peking University with the LAMOST 2D pipeline (v2.6; Luo et al. 2015) and the flux calibration pipeline developed specifically for the LSS-GAC Xiang et al. 2015c).

\subsection{Examining the method with duplicate observations}

For a given target spectrum $\boldsymbol{x}_{\text {target }}$, let $d_{\mathrm{g}}$ denote the maximal value of the kernel function,

$$
d_{\mathrm{g}} \equiv k\left(\boldsymbol{x}_{\text {target }}, \boldsymbol{x}_{\text {train }}\right)=\exp \left(\frac{-\left\|\boldsymbol{x}_{\text {target }}-\boldsymbol{x}_{\text {train }}\right\|^{2}}{c}\right) .
$$

Here $\boldsymbol{x}_{\text {train }}$ represents the training spectrum and the subscript $g$ means 'Gaussian'. The $d_{\mathrm{g}}$ is a metric describing similarities between the target and training spectra. If a target spectrum is exactly the same as one of the training spectra, then $d_{\mathrm{g}}$ is unity. A small value of $d_{\mathrm{g}}$ could be due to either poor quality (mostly low SNR) of the target spectrum or the fact that the target spectrum is so special that none of the training spectra match it. If the analysis yields a small value of $d_{\mathrm{g}}$, one expects that the estimated atmospheric parameters are of low accuracy. As an example, Fig. 10 plots the estimated $T_{\text {eff }}$ for the LSS-GAC stars using the MILES as the training set as a function of $d_{\mathrm{g}}$. The Figure shows that as $d_{\mathrm{g}}$ diminishes to 0 , the estimated $T_{\text {eff }}$ converages to a specific value around $4600 \mathrm{~K}$. Similar artifacts are also found in the case of $\log g$ and $[\mathrm{Fe} / \mathrm{H}]$ estimation. The artifacts arise mainly from defects in the spectra, especially at low spectral SNRs. The bottom panel of Fig. 10 illustrates that the $d_{\mathrm{g}}$ value is very sensitive to spectral SNR. A small

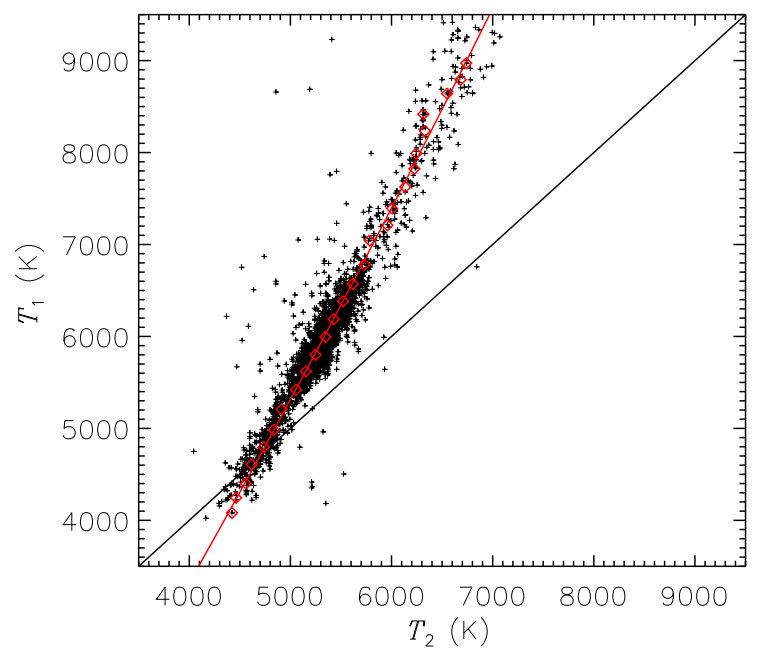

Figure 11. Comparison of effective temperatures deduced from spectra that yield $0.5<d_{\mathrm{g}}<0.55\left(T_{2}\right)$ with their counterparts derived from duplicate observations of $d_{\mathrm{g}}>0.9\left(T_{1}\right)$. The red diamonds are mean values of $T_{2}$ in different bins of $T_{1}$, and the red line is a 2 nd-order polynomial fit to the red diamonds.

$d_{\mathrm{g}}$ value (e.g. $\left.<0.5\right)$ is most likely from spectra of SNR lower than 20. Since there are a large number of spectra of low SNRs in the LAMOST archive, it is very important to overcome those artifacts in order to avoid dramatic systematic bias in the estimated parameters.

Fortunately, in our sample there are a substantial number of stars with duplicate observations that yield different values of $d_{\mathrm{g}}$. This allows us to carry out an internal calibration to correct for the artifacts for small values of $d_{\mathrm{g}}$. In doing so, we search for stars with duplicate spectra by requiring that one yields $d_{\mathrm{g}}>0.9$ whereas the other has $d_{\mathrm{g}}<0.9$. In total, 143,788 pairs of duplicate spectra are found. Parameters deduced from spectra that yield $d_{\mathrm{g}}>0.9$ are supposed to be free from the artifacts. Results derived from spectra that yield $d_{\mathrm{g}}<0.9$ are grouped into bins of $d_{\mathrm{g}}$ with a bin size of 0.05. For each bin, parameters derived from the duplicate spectra sets are fitted by polynomials, and the resultant polynomials are used to calibrate parameter values derived from spectra of small $d_{\mathrm{g}}$ to those deduced from spectra of $d_{\mathrm{g}}>0.9$. Here we adopt a 2nd-order polynomial for the calibration of effective temperature, and linear function for the calibration of other parameters. As an example, Fig. 11 plots the polynomial fit of $T_{\text {eff }}$ deduced from the duplicate spectra sets for the case that the lower quality set have $0.5<d_{\mathrm{g}}<0.55$. The Figure shows that a 2 nd-order polynomial fit of $T_{1}$ as a function of $T_{2}$ is sufficient. Here $T_{1}$ represents $T_{\text {eff }}$ derived from the set of spectra with $d_{\mathrm{g}}>0.9$, and $T_{2}$ represents those derived from the other set of spectra with $0.5<d_{\mathrm{g}}<0.55$. Note that Fig. 11 also shows a few outliers. For instance, there are several stars with $T_{1}>7500 \mathrm{~K}$ and $T_{2}<6000 \mathrm{~K}$. For those stars, LSP3 parameters deduced from the duplicate spectra also exhibit significant scatter. Further inspection shows that the duplicate spectra of those stars do vary significantly, indicating that they are indeed variables.

In the following subsections, we will not repeat the introduction of the corrections for systematic bias of parameters for low values of $d_{\mathrm{g}}$. All results presented refer to those after the internal calibrations. In addition, parameters deduced from spectra with 


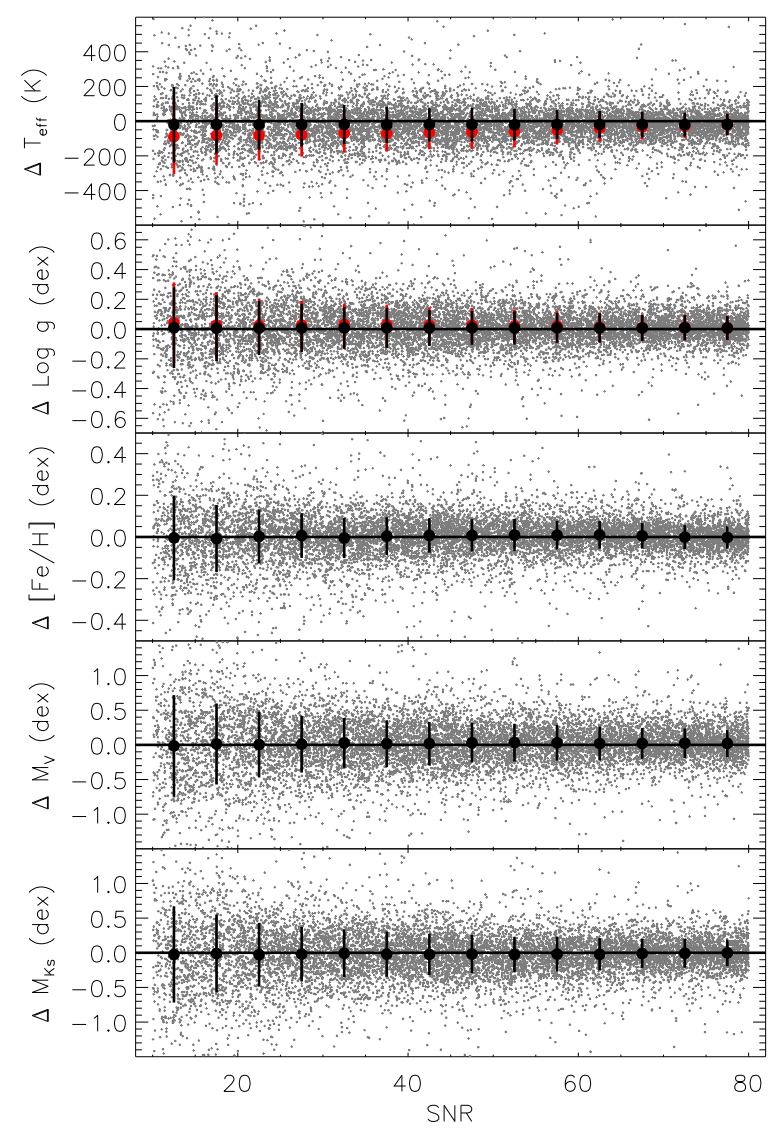

Figure 12. Differences of atmospheric parameters and absolute magnitudes derived from duplicate observations of different spectral SNR, plotted against the SNR of lower-quality observation. The SNR of the higher quality spectrum is required to be larger than 80 per pixel. Parameter values derived from the lower-quality set of duplicate observations have been internally re-calibrated accounting for small values of $d_{\mathrm{g}}$ (see text for detail) before the differences are calculated. The red and black dots are the mean differences before and after a correction for the trend with SNR, respectively. The error bars are the standard deviations for stars in the individual bins of the SNR

$d_{\mathrm{g}}<0.2$ are discarded due to potentially large uncertainties of the internal calibrations.

\subsection{Stellar atmospheric parameters derived with the MILES library}

As introduced in $\$ 4.1$, we estimate KPCA effective temperature and metallicity for all stars of $T_{\mathrm{eff}}^{\mathrm{LSP} 3}<12000 \mathrm{~K}$ and surface gravity for stars of $\log g_{\mathrm{LSP} 3}>3.0$ dex utilizing the MILES training stars. The resultant parameters are examined detailedly by comparing results from duplicate observations and comparing with external data sets.

\subsubsection{Random errors induced by imperfections of the spectra}

Fig. 12 plots the differences of atmospheric parameters deduced from duplicate observations as a function of the lower SNR. As default, systematics in parameters of stars with low $d_{\mathrm{g}}$ values have been corrected as described above. Here the duplicate observations refer to those carried out in different nights. The Figure shows that
$T_{\text {eff }}$ deduced from spectra of low SNR are slightly underestimated compared to those for high spectral SNR, and the underestimation reaches about $100 \mathrm{~K}$ at a SNR of $10 . \log g$ of stars with SNR $\sim 10$ exhibit an overestimation of $\sim 0.05$ dex compared to their high SNR counterparts. For $[\mathrm{Fe} / \mathrm{H}]$, there are no significant trends with SNR. We therefore further correct for the systematics in $T_{\text {eff }}$ and $\log g$ induced by low spectral SNR via linearly interpolating the mean differences as a function of SNR. The Figure also shows that the dispersions, which indicate random errors of the parameters induced by imperfections of the spectra, are a sensitive function of the spectral SNR, and become very small at high SNR.

To further investigate random errors of the deduced parameters induced by imperfections of the spectra, we select duplicate stars that have comparable (within 20 per cent of each other) spectral SNR and are collected in different nights, and group them into bins of $\mathrm{SNR}, T_{\text {eff }}$ and $[\mathrm{Fe} / \mathrm{H}]$. In each bin, a resistant estimate of the standard deviation of the differences of parameter values derived from the duplicate observations is calculated. The standard deviations, after divided by the square root of 2 , is adopted as the random errors induced by imperfections of the spectra. Fig. 13 shows that the random errors are a sensitive function of the spectral SNR, and also vary moderately with the spectral type $\left(T_{\text {eff }}\right)$. For a SNR of 20, the random error of $T_{\text {eff }}$ is $100-120 \mathrm{~K}$ for stars of $T_{\text {eff }}<7000 \mathrm{~K}$, and about $200 \mathrm{~K}$ for stars of $T_{\text {eff }}>7000 \mathrm{~K}$. For a higher SNR of 50 , the random error of $T_{\text {eff }}$ decreases to $\sim 50 \mathrm{~K}$ for cool stars, and to $\sim 100 \mathrm{~K}$ for those hot ones. No significant variations in the random errors are seen for stars of different $[\mathrm{Fe} / \mathrm{H}]$. For $\log g$, the random error is about 0.15 dex for a SNR of 20, and decreases to 0.05 dex at high SNRs, without significant variations with $T_{\text {eff }}$ or $[\mathrm{Fe} / \mathrm{H}]$. The random error of $[\mathrm{Fe} / \mathrm{H}]$ is close to 0.1 dex for a SNR of 20 for stars of $T_{\text {eff }}<7000 \mathrm{~K}$, and decreases to $\sim 0.05$ dex given high SNR. While for hot $\left(T_{\text {eff }}>7000 \mathrm{~K}\right)$ or metal-poor stars, the random errors amount to $0.15-0.20$ dex for a SNR of 20 . Note that the above random errors are not realistic errors of the estimated parameters, but refers to only errors induced by uncertainties in the observed spectra. The realisitic errors should also include those induced by the method of analysis, which can be approximated by the regression residuals of the training set, as well as potential systematic bias in the MILES stellar atmospheric parameters, which will be discussed in the following subsections.

\subsubsection{Comparing $[\mathrm{Fe} / \mathrm{H}]$ with high-resolution spectroscopy}

We compare our $[\mathrm{Fe} / \mathrm{H}]$ estimates with two external high-resolution spectroscopic data sets: the PASTEL catalog and the catalog of Huber et al. (2014). The PASTEL catalog (Soubiran et al. 2010) is a collection of literature determinations of stellar atmospheric parameters for 16649 stars, about 6000 of which have $[\mathrm{Fe} / \mathrm{H}]$ determinations from high-resolution spectroscopy. The catalog of Huber et al. (2014) collects stellar parameters from the recent literatures published in recent years for stars in the Kepler field. The catalog contains 819 stars whose $[\mathrm{Fe} / \mathrm{H}]$ are deduced from highresolution spectroscopy. For the comparison, we exclude stars with a LAMOST spectral SNR lower than 20, as well as stars whose $[\mathrm{Fe} / \mathrm{H}]$ in the PASTEL catalog are from literatures published before 1990. After the cuts, we have a sample of 150 stars in common with the PASTEL catalog, and a sample of 239 stars in common with the catalog of Huber et al.

Fig. 14 plots the differences between our $[\mathrm{Fe} / \mathrm{H}]$ estimates and the high-resolution results against $T_{\text {eff }}, \log g$ and $[\mathrm{Fe} / \mathrm{H}]$. A resistant estimate of the mean and standard deviation for the overall sample of stars in common with the PASTEL catalog are -0.01 dex 

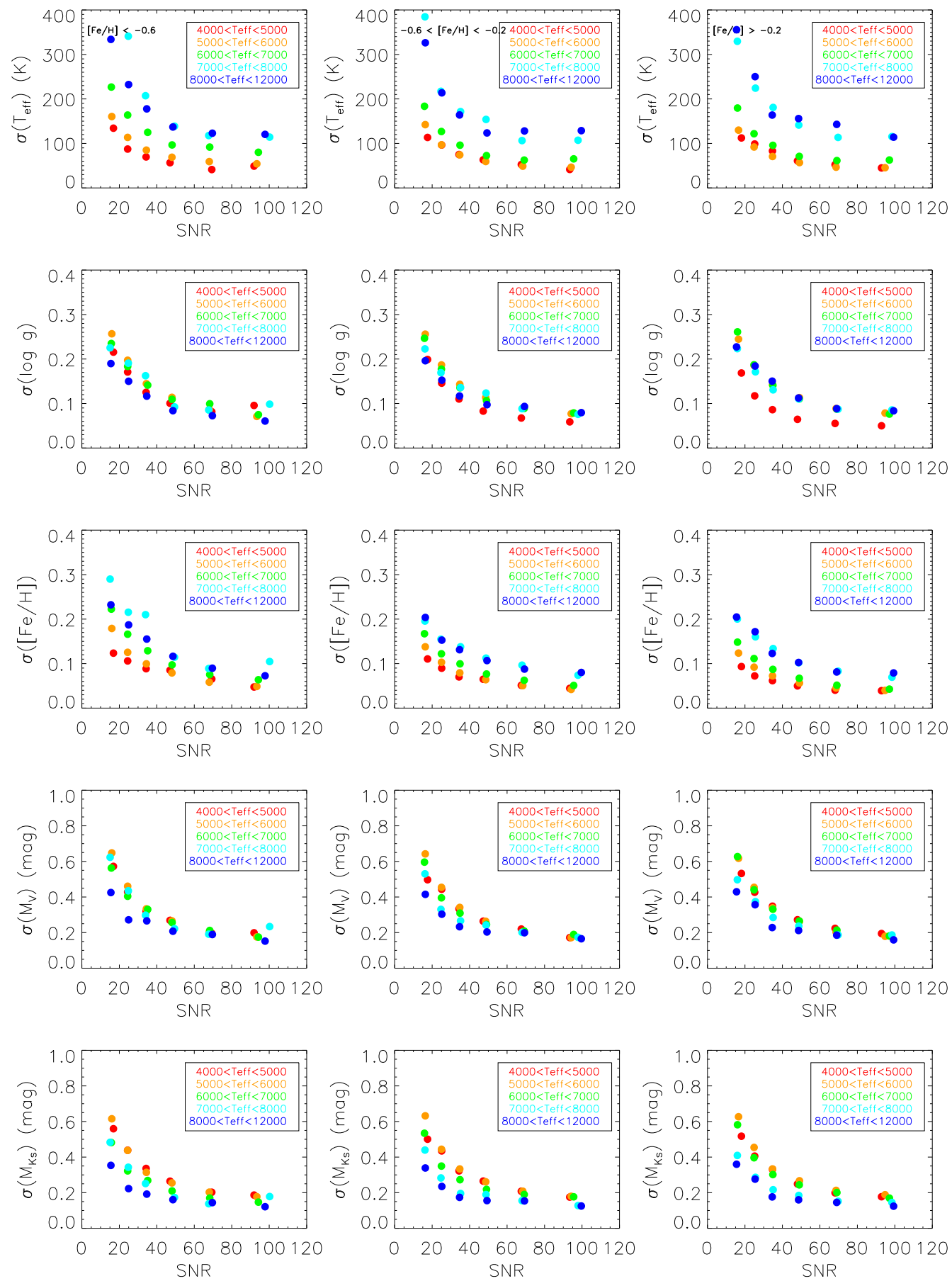

Figure 13. Random errors of the estimated parameters, estimated by comparing results deduced from duplicate observations of comparable SNRs, as a function of the spectral SNR. Stars are grouped into different bins of $T_{\text {eff }}$ (colour-coded) and [Fe/H] (different columns).

and 0.14 dex, and those values are -0.03 dex and 0.13 dex for stars in common with the catalog of Huber et al. The differences are not homogeneously distributed across the parameter space. For both samples, the dispersions for stars of $T_{\text {eff }}>7000 \mathrm{~K}$ are significantly larger than those for the bulk, dominated by F/G/K stars of $4000<T_{\text {eff }}<7000 \mathrm{~K}$. In fact, a Gaussian fit to the distribution of the differences yields a dispersion, which reflects value for the bulk stars, of 0.10 dex only for both samples. Note that $[\mathrm{Fe} / \mathrm{H}]$ uncertainties of the high-resolution results themselves are expected to be at 0.1 dex level. No significant systematic trends are seen in the data, but there are several outliers showing very large deviations from the high resolution determinations. For those outliers, it is found that their literature values of $T_{\text {eff }}$ differ significantly with our estimates, but our values are consistent with results from LSP3. A 

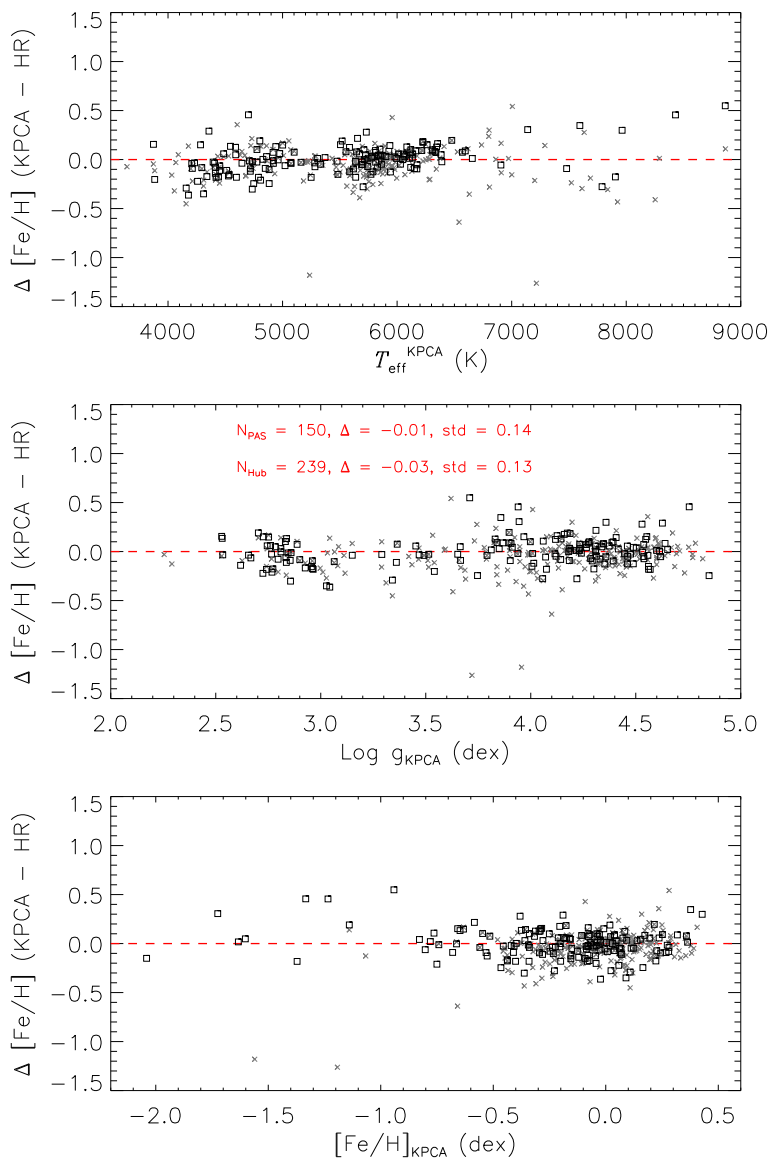

Figure 14. Differences between $[\mathrm{Fe} / \mathrm{H}]$ estimated by KPCA utilizing the MILES training set and values of high-resolution spectroscopic measurements, plotted against the KPCA atmospheric parameters. Squares and crosses represent high-resolution results from the PASTEL catalog and Huber et al. 2014), respectively. The number of stars in common with the two catalogues, as well as values from a resistant estimate of the mean and standard deviation of the differences, are marked in the middle panel of the plot.

further inspection of LAMOST spectra for those stars corroborates the robustness of our determinations.

\subsubsection{Comparison with photometric temperatures}

We compare our estimates of effective temperature with photometric temperature yielded by the metallicity-dependent colourtemperature relation of Huang et al. (2015a). The relation is derived based on more than a hundred nearby stars with direct effective temperature measurements, and are applicable for stars of $[\mathrm{Fe} / \mathrm{H}]>-1.0$ dex. The de-reddened $g-K_{\mathrm{s}}$ colour and the above estimated $[\mathrm{Fe} / \mathrm{H}]$ are used to generate photometric temperatures for our sample stars. Here the SDSS $g$-band photometry is from the XSTPS-GAC survey (Zhang et al. 2014; Liu et al. 2014) for stars of $g>13.5$, and from the APASS survey (Henden \& Munari 2014) for the brighter stars, while the $K_{\mathrm{S}}$ - band photometry is from the 2MASS catalog (Skrutskie et al. 2006). The $g-K_{s}$ colour is dereddened with interstellar reddening $E(B-V)$ from the map of Schlegel, Finkbeiner \& Davis (1998, hereafter SFD) and extinction coefficients from Yuan, Liu \& Xiang (2013). To reduce errors from
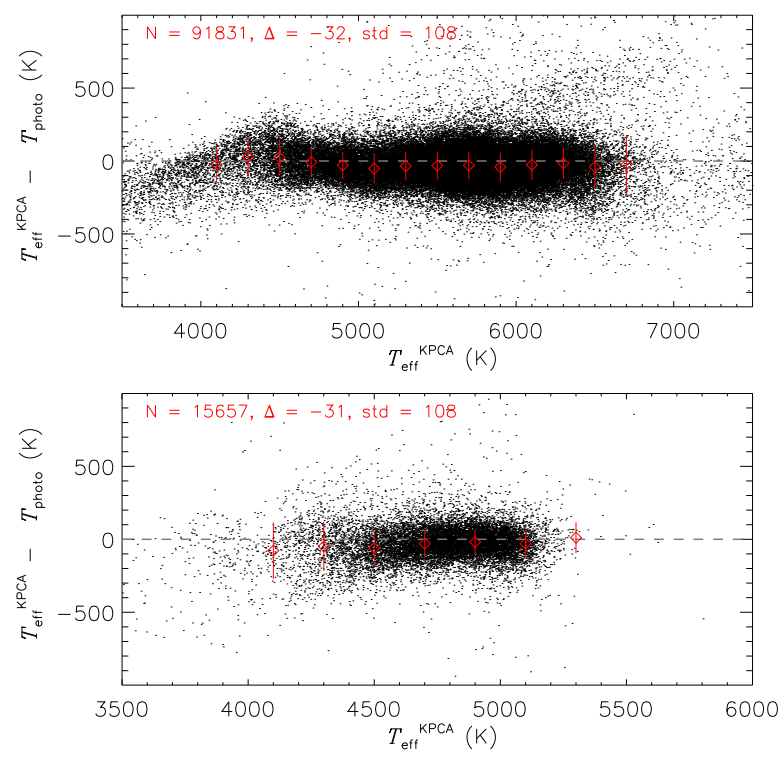

Figure 15. Differences between the estimated effective temperature $T_{\mathrm{eff}}^{\mathrm{KPCA}}$ with photometric temperature $T_{\text {photo }}$ deduced from the metallicity-dependent colour-temperature relation of Huang et al. (2015a) for dwarf stars (upper) and giant stars (lower). The red diamonds and error bars are the mean differences and standard deviations at different bins of $T_{\mathrm{eff}}^{\mathrm{KPCA}}$. The number of stars, as well as a resistant estimate of the overall mean and standard deviation of the differences, are marked in the plot.

uncertainties in interstellar extinction, we select stars of Galactic latitude $|b|>10^{\circ}$ and $E(B-V)_{\mathrm{SFD}}<0.05$ mag only for the determination of photometric temperatures. To ensure high accuracy of the photometric data, we further require the stars have a magnitude of $10.5<g<18.5 \mathrm{mag}, 6<K_{\mathrm{S}}<15.5 \mathrm{mag}$, and magnitude errors smaller than 0.05 mag in both bands. For the comparison, we also require the stars to have a spectral SNR $>20$ and $[\mathrm{Fe} / \mathrm{H}]>-1.0$ dex. The above criteria lead to 91831 dwarf stars $(\log g>3.8 \mathrm{dex})$ and 15657 giant stars $(\log g<3.5 \mathrm{dex})$ in our sample.

Fig. 15 plots the differences between our estimates of effective temperatures $T_{\mathrm{eff}}^{\mathrm{KPCA}}$ and the photometric estimates $T_{\text {photo }}$ as a function of the former. The mean and standard deviation of the differences are -32 and $108 \mathrm{~K}$, respectively, for dwarf stars of $3500<T_{\mathrm{eff}}^{\mathrm{KPCA}}<7500 \mathrm{~K}$. No obvious trend of differences is seen in the temperature range $4000-7000 \mathrm{~K}$. Note that the uncertainties of photometric temperatures are believed to be about 2 per cent (Huang et al. 2015a). The number of hot ( $\left.T_{\mathrm{eff}}^{\mathrm{KPCA}}>7000 \mathrm{~K}\right)$ stars are too small so that we do not discuss those stars here. For stars cooler than $3800 \mathrm{~K}$, our estimates maybe not reliable due to a lack of training stars. For giant stars, the differences have a mean and standard deviation of -31 and $108 \mathrm{~K}$, respectively. Again, no significant trend of differences with temperature is seen, except for stars with extremely temperatures $\left(T_{\mathrm{eff}}^{\mathrm{KPCA}}<4500 \mathrm{~K}\right)$, foe which our estimates seem to be lower than the photometric estimates by a few tens Kelvin.

\subsubsection{Uncertainties in the surface gravity}

In Fig. 1, we show that given a PC number of 100, our estimates of $\log g$ from the LAMOST spectra are consistent well with the asteroseismic measurements for the LAMOST-Kepler common stars, 
with a mean difference and standard deviation of only $0.00 \mathrm{dex}$ and 0.11 dex, respectively, suggesting that our $\log g$ estimates have achieved a precision of 0.1 dex. However, this is only realistic for stars with high spectral SNRs because most of the LAMOSTKepler stars have a spectral SNR higher than 50. Considering the random errors of $\log g$ are sensitive to SNR as illustrated by Fig. 13, we expect that uncertainties of the $\log g$ estimates increase to 0.2 dex for a SNR of 20. Note that here the asteroseismic sample contains only stars of $5500<T_{\text {eff }}^{\mathrm{KPCA}}<6500 \mathrm{~K}$, thus $\log g$ estimates for stars of temperatures outside this range need to be further examined. It is expected that $\log g$ estimates for stars of higher temperatures have larger uncertainties because, as Fig. 2 demonstrates, the method errors become significantly larger at higher temperatures.

We make a further sanity check of $\log g$ estimates with member star candidates of open cluster M 67, which has a literature metallicity of -0.01 dex (Jacobson, Pilachowski \& Friel 2011) and an age of $4.3 \mathrm{Gyr}$ (Richer et al. 1998; Salaris, Weiss \& Percival 2004). Specific observations are designed to target member candidates of open clusters utilizing test observation nights of the LAMOST (Yang et al., in preparation), and we select the cluster member stars based on the spatial position, the Hertzsprung-Russel (HR) diagram and the radial velocity yielded by the LSP3 in the same way as Xiang et al. (2015a). In total, more than 500 member star candidates of M67 are selected. Here for the sanity check we pick out stars with a SNR higher than 20 and a $\log g$ yielded by the LSP3 template matching method larger than 3.0 dex. These criteria lead to 251 unique member candidates, whose distribution in the $T_{\text {eff }}-$ $\log g$ plane are plotted in the left panel of Fig. 16. Also plotted in the Figure are three Yonsei-Yale $\left(\mathrm{Y}^{2}\right)$ isochrones (Demarque et al. 2004) with solar metallicity and ages of 3, 4 and 5 Gyr, respectively. The Figure illustrates that though there are some outliers, the main sequence and the main sequence turn-off stars are consistent well with the isochrones of $4 \mathrm{Gyr}$, while $\log g$ of the bulk of subgiant stars are lower than the isochrone values by about 0.1 dex.

\subsection{Absolute magnitudes estimated with the LAMOST-Hipparcos training set}

Absolute magnitudes in $V$ and $K_{\mathrm{s}}$ bands for all LSS-GAC stars of $T_{\mathrm{eff}}^{\mathrm{LSP} 3}<12000 \mathrm{~K}$ are derived with the LAMOST-Hipparcos training data set. Detailed internal examinations and calibrations are first carried out as introduced in $\$ 5.1$.

The bottom panels of Fig. 12 illustrate that though low spectral SNR induces larger random errors, it does not cause systematic bias to our results. A detailed investigation of random errors induced by spectral imperfections for stars of different SNRs and stellar atmospheric parameters is presented in Fig. 13. The Figure shows that the random error is a steep function of spectral SNR, and also vary with spectra types. At a SNR of 20, random errors of both $\mathrm{M}_{V}$ and $\mathrm{M}_{K \mathrm{~s}}$ vary from 0.3 to $0.5 \mathrm{mag}$, depending on effective temperatures of the stars, while at a SNR of 50, the random errors decrease to $0.15-0.3 \mathrm{mag}$, and the values continuously decrease to $0.1-0.15$ dex at high SNRs.

The middle panel of Fig. 16 shows that for member star candidates of $\mathrm{M} 67$, the $T_{\text {eff }}-\mathrm{M}_{V}$ diagram match well with the $\mathrm{Y}^{2}$ isochrones. Not only the main sequence and main sequence turnoff stars, but also the subgiant stars, are all consistent with the isochrone of $4 \mathrm{Gyr}$. The right panel of the Figure plots the distribution of the $V$-band distance modulus of M 67 derived with the individual stars. The distribution yields a mean modulus of 9.58 mag and a standard deviation of 0.33 mag. Outliers are clearly visible in

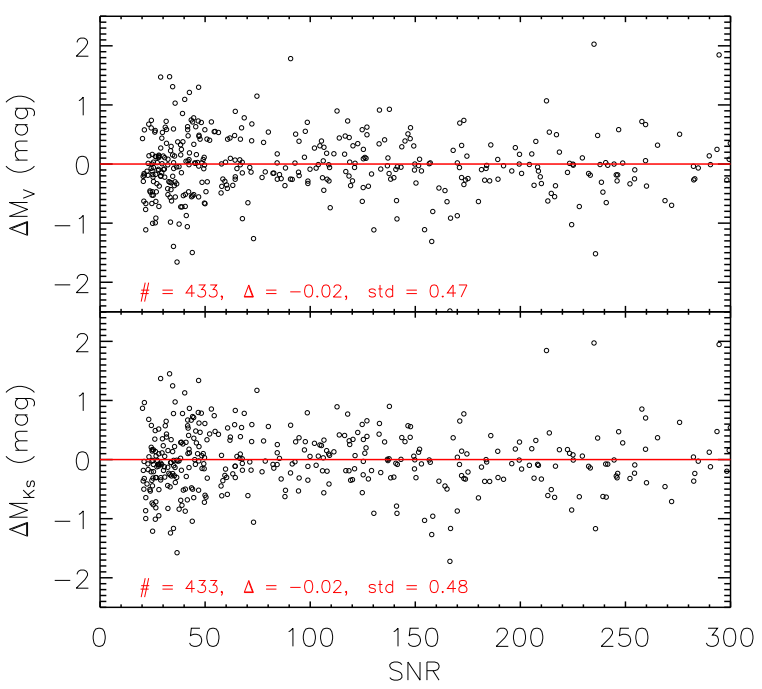

Figure 17. Differences of absolute magnitudes between with the estimated values and those deduced from the Hipparcos distance for the LAMOSTHipparcos test stars as a function of spectral SNR. The number of stars, the mean and dispersion of a Gaussian fit on the overall distribution of the differences are marked in the plot.

the Figure. Some of the outliers are expected to be binaries/multiple stars and/or variable stars. It is also possible that some of the outliers are contaminations of field stars. In fact, a Gaussian fit, which is less affected by the outliers, yields a dispersion of only $0.24 \mathrm{mag}$, corresponding to a distance error of 12 per cent.

We further utilize LAMOST-Hipparcos stars that have reliable parallax measurements but are not included in the training sample as a test data set to examine our results. Those stars are not included in the training data set due to either low spectral SNR $(<50)$ or that they are duplicate observation counterparts of the training stars. Here we select only stars with uncertainties in absolute magnitudes smaller than 0.3 mag for $\mathrm{M}_{V}$ and $\mathrm{M}_{K \mathrm{~s}}$ deduced utilizing the Hipparcos parallax, and further require that the stars have a spectral SNR higher than 20. These criteria lead to 433 stars in our test sample. Fig. 17 plots the differences of absolute magnitudes between our estimates and those deduced utilizing the Hipparcos parallax. The differences are plotted against spectral SNR. The mean and standard deviation of the overall differences are respectively $-0.02 \mathrm{mag}$ and $0.47 \mathrm{mag}$ for $\mathrm{M}_{V},-0.02$ and 0.48 mag for $\mathrm{M}_{K \mathrm{~s}}$. Considering that typical uncertainties of absolute magnitudes propagated from errors of the Hipparcos parallax of our sample stars are $0.2-0.3 \mathrm{mag}$, typical errors of our magnitude estimates should be $0.36-0.43$ mag for both $\mathrm{M}_{V}$ and $\mathrm{M}_{K \mathrm{~s}}$. Note however that, uncertainties in the estimated absolute magnitudes are sensitive to spectral SNR and spectral types. For stars of SNR higher than 50, the above standard deviation becomes 0.4 mag only, corresponding to an uncertainty of $0.3-0.4 \mathrm{mag}$ in our estimates. The Figure also shows a considerable fraction of stars with large differences (e.g. $>1.0 \mathrm{mag}$ ). By referring to the SIMBAD database (Wenger et al. 2000), many of those stars are binary/multiple or variable stars, while the others are likely caused by spectral imperfections - especially because the LAMOST-Hipparcos stars are too bright to be feasible for observation with the LAMOST. In fact, most of the LAMOST-Hipparcos stars are observed in conditions with bright lunar light, and for many of them, offsets in coordinates are set for 

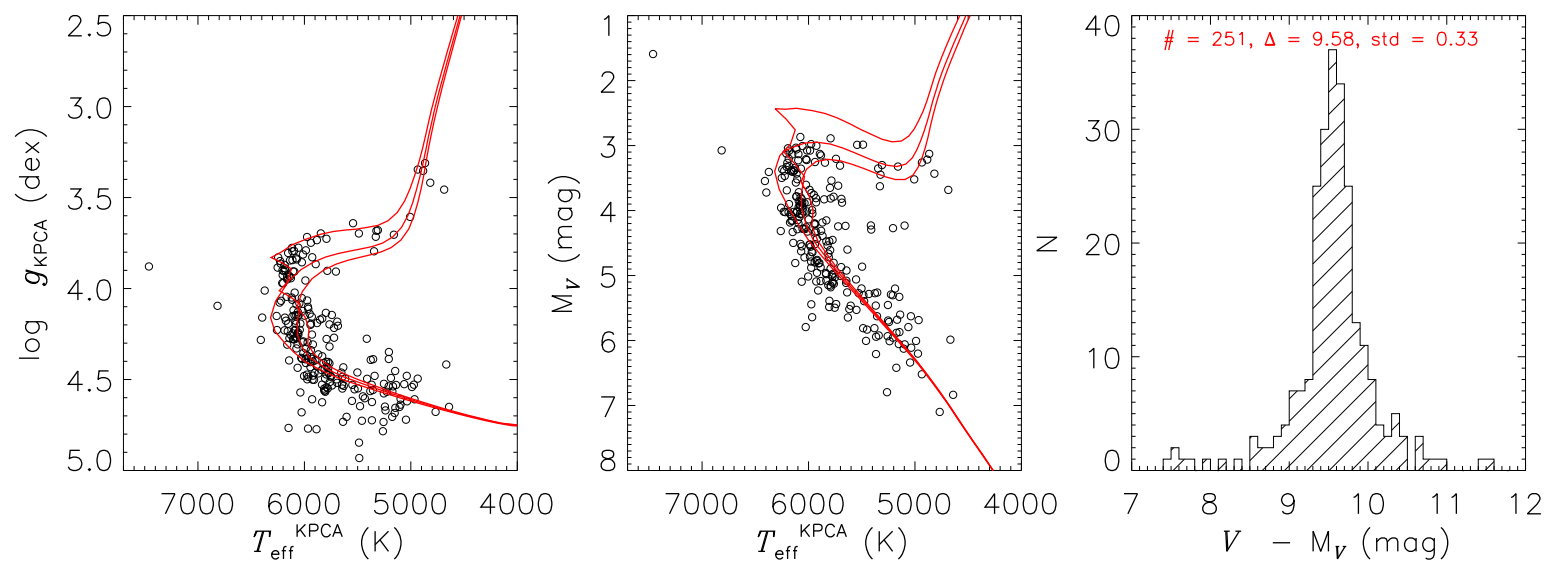

Figure 16. Distribution of member star candidates of M 67 in the $T_{\text {eff }}-\log g$ (left) and the $T_{\text {eff }}-\mathrm{M}_{V}$ diagrams. The red lines are Yonsei-Yale isochrones with solar metallicity and ages of 3,4 and $5 \mathrm{Gyr}$ from left to right, respectively. The right panel plots the histogram of distance modulus in $V$-band derived from the individual member candidates. The number of stars, mean and standard deviation of the distribution are marked.

fiber positioning to avoid saturation. As a result, for those stars, even though the reported SNRs are high, their real spectral quality are actually not good, leading to inaccurate parameter estimates.

\subsection{Surface gravities estimated with the LAMOST-Kepler training set}

Surface gravity $\log g$ for all stars of $\log g_{\mathrm{LSP} 3}<3.8 \mathrm{dex}$ and $T_{\text {eff }}^{\mathrm{LSP} 3}<5500 \mathrm{~K}$ are estimated with the KPCA regression method using the LAMOST-Kepler training set. Internal calibrations for stars of low $d_{\mathrm{g}}$ values are carried out as introduced in $\$ 5.1$. As an evaluation of $\log g$ estimates, the upper panel of Fig. 18 plots the differences between the KPCA $\log g$ and the asteroseismic values for the LAMOST-Kepler test sample (cf. §4.2) as a function of the spectral SNR. It shows that the mean differences are close to zero at different SNRs, and the dispersions (standard deviations) decrease from $\sim 0.3$ dex at a SNR of 10 to $\sim 0.2$ dex at a SNR of 30 , and $\sim 0.1$ dex at high SNRs. The results are consistent with those for the training sample, which is $-0.01 \pm 0.11$ dex (\$4.2), considering that the training sample includes only stars with SNR higher than 50. Such a precision is comparable to that of Liu et al. (2015), who estimate $\log g$ from the LAMOST spectra using a SVR method, also trained by the LAMOST-Kepler sample stars. Note that for stars with low SNRs (e.g. < 20), we find a slightly larger dispersion of differences between our $\log g$ estimates and the asteroseismic values than Liu et al. This is because we use the standard deviation to represent the dispersion, while Liu et al. adopt the $1 \sigma$ value of the Gaussian distribution.

The lower panel of Fig. 18 shows the dispersions of $\log g$ differences deduced from duplicate observations as a function of the spectral SNR. Here we have required that for each star, the duplicate observations have roughly the same SNRs, and the dispersions have been divided by the square root of 2 . The Figure shows that the dispersions decrease from $\sim 0.2$ dex at a SNR of 20 to $\sim 0.1$ dex at a SNR of 50, and further decrease to 0.05 dex at high SNRs (>100). The dependence of the dispersions on metallicity is negligible. Note that for the metal-poor stars, though the dispersions are small, it is possible that systematic bias dominate the real uncertainties since there are only a few stars of $[\mathrm{Fe} / \mathrm{H}]<-1.0$ dex in our training sample (cf. §4.2). Note also that though the dispersions at high SNRs can be as small as 0.05 dex, the real uncertainties at
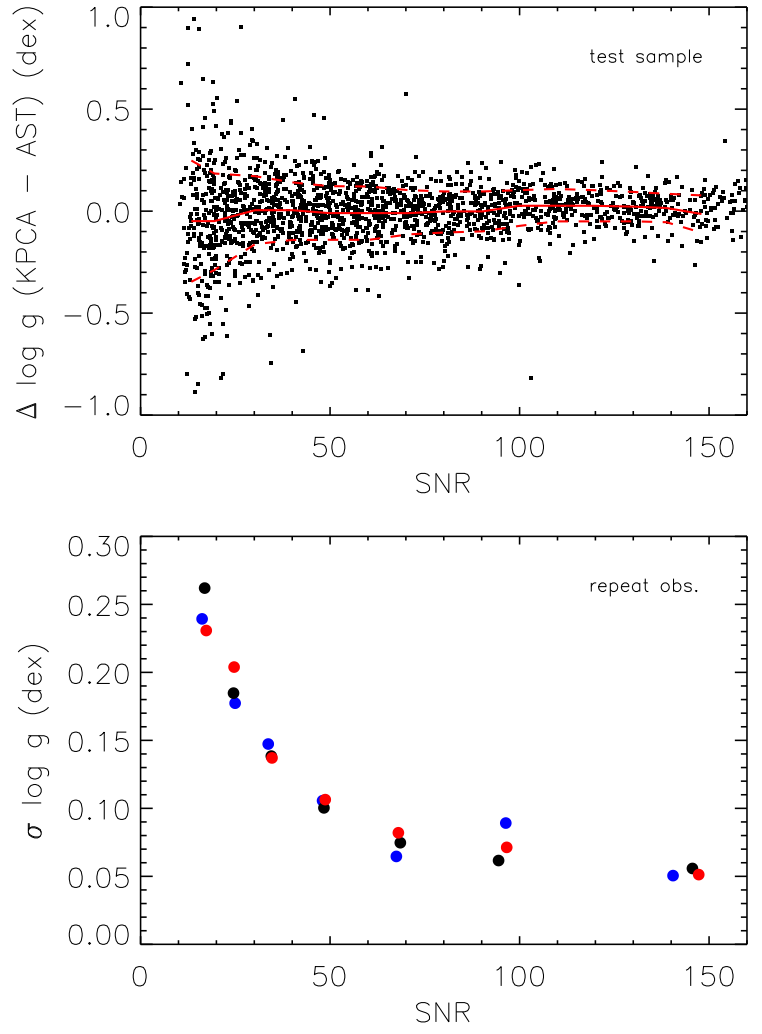

Figure 18. Upper panel: Differences of the estimated $\log g$ with the asteroseismic values for the LAMOST-Kepler test star sample; Lower panel: Random errors of $\log g$ deduced by comparing results of duplicate observations, as a function of the SNR. Different colours represent results for stars in different ranges of $[\mathrm{Fe} / \mathrm{H}]$ (blue: $[-3.0,-0.6]$; black: $[-0.6,-0.2]$, red: $[-0.2,0.5])$.

such SNRs are however, larger than the dispersions. This is because the dispersions deduced from duplicate observations only account for uncertainties induced by imperfections of the LAMOST spectra but not include uncertainties of the method itself. 
Since the dispersions deduced from the LAMOST-Kepler test sample are contributed by both imperfections of the LAMOST spectra and inadequacy of the method, they are used to estimate realistic errors of $\log g$ estimates. Errors of $\log g$ are assigned to individual stars based on the spectral SNRs. It seems quite clear that uncertainties in the KPCA $\log g$ estimates are only $\sim 0.1$ dex at high SNRs ( $\gtrsim 80)$.

\subsection{Metallicity and elemental abundances estimated with the LAMOST-APOGEE training set}

Metallicity $[\mathrm{M} / \mathrm{H}], \alpha$-element to metal abundance ratio $[\alpha / \mathrm{M}], \alpha$ element to iron abundance ratio $[\alpha / \mathrm{Fe}]$ and elemental abundances $[\mathrm{C} / \mathrm{H}],[\mathrm{N} / \mathrm{H}]$ and $[\mathrm{Fe} / \mathrm{H}]$ are estimated for LSS-GAC stars of $\log g_{\mathrm{LSP} 3}<3.8 \mathrm{dex}$ and $T_{\mathrm{eff}}^{\mathrm{LSP} 3}<5500 \mathrm{~K}$ utilizing the LAMOSTAPOGEE training stars, and internal calibrations for stars of low $d_{\mathrm{g}}$ values are carried out as introduced in $§ 5.1$.

To examine precisions of the estimated abundances, Fig. 19 plots the differences between our estimated values and the APOGEE measurements as a function of LAMOST spectral SNR for the test star sample defined in $\S 4.4$. The Figure shows that the mean differences at all SNR are close to zero for all abundances, and the dispersions decrease significantly with increasing SNR at the lower SNR side, while at the higher SNR side, the dispersions keep almost flat. Such a trend of dispersions is expected, because for stars with low spectral SNR, uncertainties of abundance estimates are dominated by random errors induced by spectral imperfections, which is a steep function of SNR, while for stars with high spectral SNR, uncertainties of abundance estimates are dominated by method errors, which are mainly propagated from uncertainties in the APOGEE measurements of the training stars. The three numbers marked in the plot are values of dispersions (standard deviations) calculated at a SNR of 20,30 and 50, respectively. They show that at a SNR of 20, dispersions of the differences for metal abundance $[\mathrm{M} / \mathrm{H}]$ and elemental abundance $[\mathrm{C} / \mathrm{H}],[\mathrm{N} / \mathrm{H}]$ and $[\mathrm{Fe} / \mathrm{H}]$ are about $0.13-0.17 \mathrm{dex}$. While those numbers decrease to $0.10-0.13 \mathrm{dex}$ at a SNR of 30 , and further decrease to 0.07 $-0.10 \mathrm{dex}$ at a SNR of 50 . For $[\alpha / \mathrm{M}]([\alpha / \mathrm{Fe}])$, the dispersions decrease from $0.06 \mathrm{dex}$ at a SNR of 20 to $0.03 \mathrm{dex}(0.04 \mathrm{dex})$ at a SNR of 50. Note that both errors in our abundance estimates and those in the APOGEE measurements have contributed to the dispersions. For stars with high spectral SNR ( $>50$ ), we expect that precision of our estimated metallicity and elemental abundances are comparable to those of the APOGEE measurements, which are $0.05-$ 0.10 dex for $[\mathrm{X} / \mathrm{H}]$, and better than $0.05 \operatorname{dex}$ for $[\alpha / \mathrm{M}]$ and $[\alpha / \mathrm{Fe}]$. Nevertheless, since the APOGEE abundances are determined with $\chi^{2}$-based algorithms and are not externally calibrated to standard scales, potential systematic biases could be hided in the APOGEE results, which must have been propagated into our estimates via the training data set. Potential systematic biases are expected to be 0.1 -0.2 dex (Holtzman et al. 2015).

Fig. 20 plots the density distribution of LSS-GAC stars with Galactic latitude $|b|>30^{\circ}$ and SNR $>30$ in the [X/Fe] (and $[\alpha / \mathrm{M}])$ against $[\mathrm{Fe} / \mathrm{H}]$ plane. For all the elements, morphology of the $[\mathrm{X} / \mathrm{Fe}]-[\mathrm{Fe} / \mathrm{H}]$ diagram are resemble to those of the APOGEE stars as shown in Fig. 14 of Holtzman et al. (2015). For the $\alpha$ element to metal abundance ratio $[\alpha / \mathrm{M}]$, as well as the $\alpha$-element to iron abundance ratios $[\alpha / \mathrm{Fe}]$, there is a decreasing trend with $[\mathrm{Fe} / \mathrm{H}]$, which means that the $\alpha$-element abundances are enhanced for metal-poor stars. There seems to be also two bulk of stars in $[\alpha / \mathrm{M}](\alpha / \mathrm{Fe})$ for a $[\mathrm{Fe} / \mathrm{H}]$ around -0.5 dex. Those trends and features are well consistent with previous results from high resolution
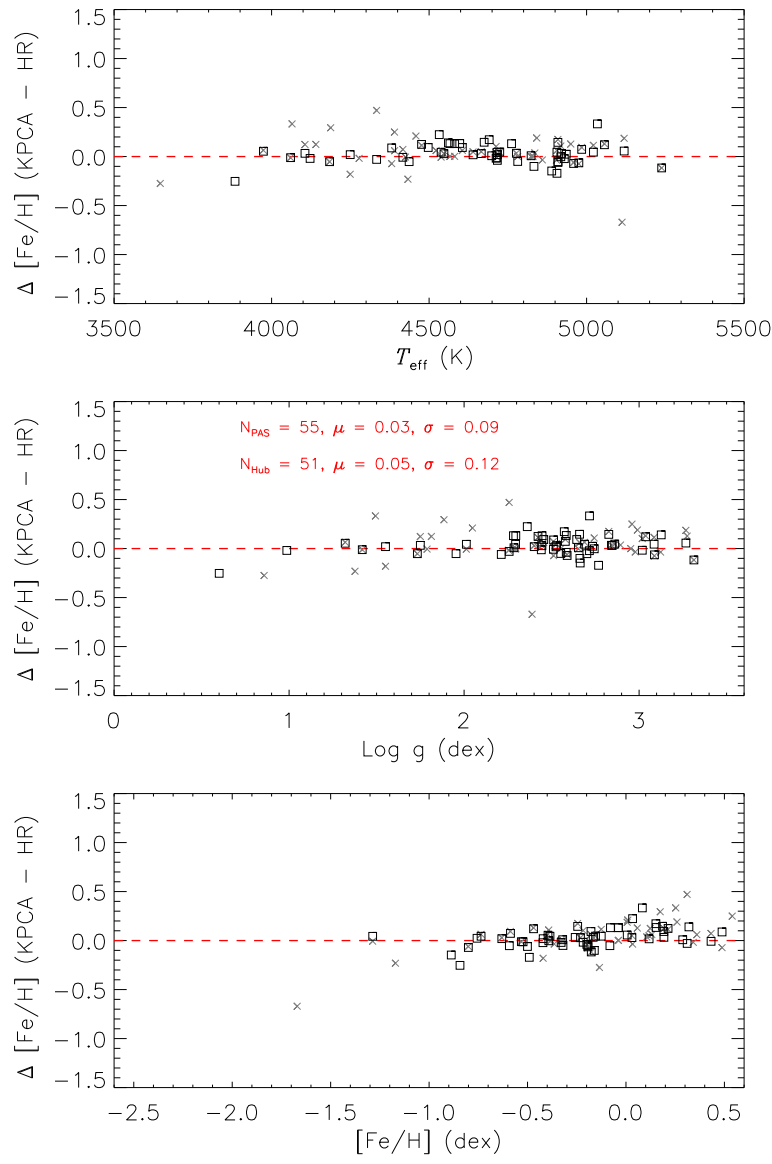

Figure 21. Differences between the $[\mathrm{Fe} / \mathrm{H}]$ estimates utilizing the LAMOST-APOGEE training stars and those of high-resolution spectroscopic measurements. The squares represent high-resolution results from the PASTEL catalog, while the crosses are from the catalog of Huber et al. (2014). The differences are shown as a function of the estimated atmospheric parameters. The number of stars for each data set, as well as a resistant estimate of the mean and standard deviation, are marked in the middle panel.

spectroscopy for stars near the solar neighbourhood. The decreasing trend of $[\alpha / \mathrm{Fe}]$ with $[\mathrm{Fe} / \mathrm{H}]$ is a consequence of Galactic chemical evolution, and the two bulk of stars are likely corresponding to the widely investigated thin and thick disk star sequences. Note that if we plot all stars in our sample rather than stars of $|b|>30^{\circ}$, a clear discrimination of the two bulks will not be visible, simply because the thin disk sequence dominates our total star sample. Nevertheless, as described in $\$ 4.4$, our elemental abundances are probably systematically overestimated for very metal-poor stars $([\mathrm{M} / \mathrm{H}]<-2.0$ dex$)$ due to a lack of metal-poor training stars, resulting a cut off of $[\mathrm{Fe} / \mathrm{H}]$ around -1.8 dex in Fig. 20, as well as a steeper decreasing trend of $[\mathrm{N} / \mathrm{Fe}]$ with $[\mathrm{Fe} / \mathrm{H}]$ in the metal-poor side.

Fig. 21 shows an external comparison of the estimated [M/H] with high-resolution spectroscopic measurements of $[\mathrm{Fe} / \mathrm{H}]$ from the PASTEL catalog and the catalog of Huber et al. (2014). Here we compare the $[\mathrm{M} / \mathrm{H}]$ but not the $[\mathrm{Fe} / \mathrm{H}]$ with high resolution spectroscopy because the $[\mathrm{M} / \mathrm{H}]$ of APOGEE stars have been externally calibrated to $[\mathrm{Fe} / \mathrm{H}]$ of star clusters, while the $[\mathrm{Fe} / \mathrm{H}]$ of APOGEE stars are not externally calibrated. In the Figure, we use the tab 

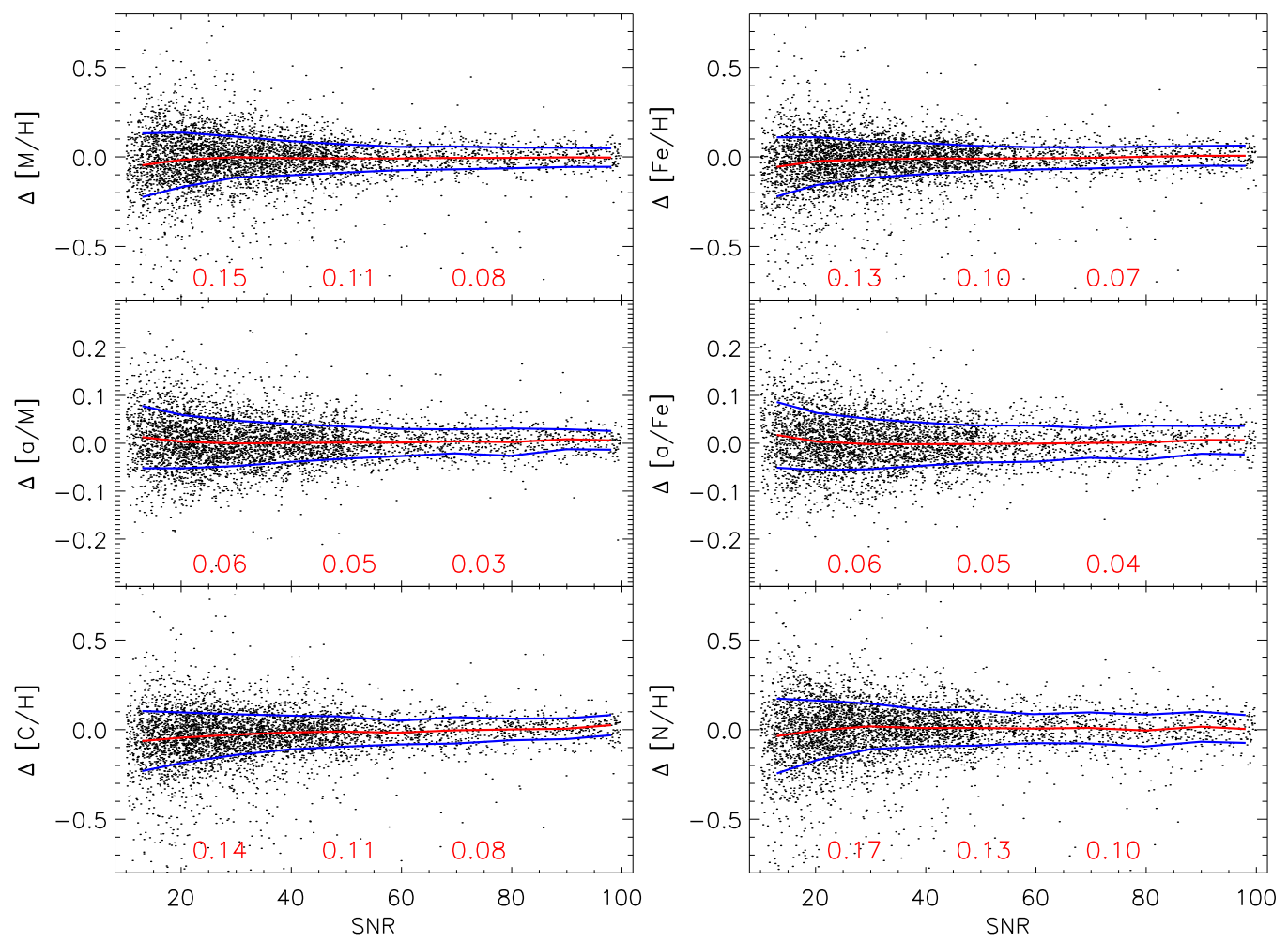

Figure 19. Differences of metallicity and elemental abundances between the KPCA estimates from the LAMOST spectra and those of the APOGEE measurements for the test star sample, as a function of spectral SNR. The mean and standard deviation of the differences as a function of SNR are plotted in red and blue solid lines, respectively. In each panel, the three numbers labeled are standard deviations calculated at a SNR of 20, 30 and 50, respectively.
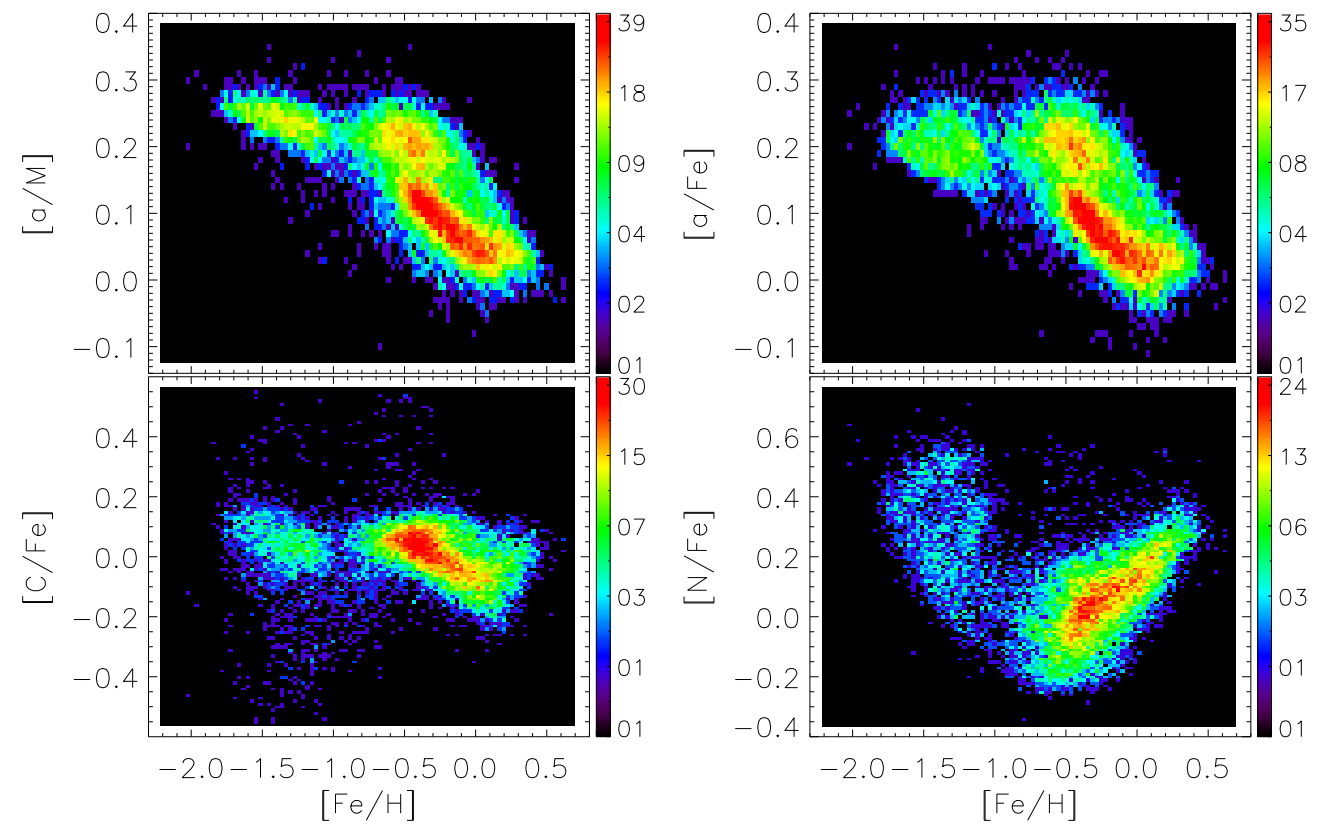

Figure 20. Colour-coded stellar number density distributions of LSS-GAC giant stars in the $[\alpha / \mathrm{M}]-[\mathrm{Fe} / \mathrm{H}]$ and $[\mathrm{X} / \mathrm{Fe}]-[\mathrm{Fe} / \mathrm{H}]$ planes. Colours indicate stellar number density in logarithmic scale. A bin size of 0.03 by 0.01 dex is adopted to generate the stellar number density distributions. Only stars of Galactic latitudes $|b|>30^{\circ}$ are shown in the plots. 
$[\mathrm{Fe} / \mathrm{H}]$ to replace $[\mathrm{M} / \mathrm{H}]$ for consistency. The Figure shows that the overall mean differences are only 0.03 and 0.05 dex for the two high-resolution spectroscopy samples, with a standard deviation of 0.09 and 0.12 dex, respectively. The small positive mean differences are contributed by stars of super-solar metallicities, for which our estimates are systematically higher than the high-resolution spectroscopic data sets. No significant biases with $T_{\text {eff }}$ and $\log g$ are seen.

Finally, according to the comparisons of metallicity and abundances with the APOGEE measurements for the test star sample (Fig. 19), we assign parameter errors to individual stars based on their spectral SNR.

\subsection{Comparison with the weighted-mean parameters of the LSP3}

Fig. 22 shows the comparisons of the estimated parameters with those derived with the $\chi^{2}$-based weighted-mean method of the LSP3 for LSS-GAC DR1. Stars with a spectral SNR lower than 20 are used for the comparison. The Figure shows that on the whole, the KPCA effective temperatures are consistent well with the LSP3 values. The mean difference is only $-42 \mathrm{~K}$, and the standard deviation for the overall stars is $153 \mathrm{~K}$. However, the differences show moderate trend with the temperature. At the higher temperature end $\left(T_{\text {eff }}^{\mathrm{LSP} 3}>7000 \mathrm{~K}\right)$, the KPCA estimates are systematically lower than the LSP3 values by about $100-200 \mathrm{~K}$. Those lower temperature estimates are the main causes of the $-40 \mathrm{~K}$ mean difference, and they also contributed a significant part of the standard deviation. In fact, for $T_{\mathrm{eff}}^{\mathrm{LSP} 3}<7000 \mathrm{~K}$, where the systematic difference is negligible, value of the overall standard deviation is only $130 \mathrm{~K}$. Since there are few stars with temperatures higher than $7000 \mathrm{~K}$ in the photometric sample for examination (cf. Fig. 15), it is not straightforward to understand the systematic difference. It is probably that for those hot stars, the KPCA effective temperature are underestimated due to a yet not fully understood reason. Regardless of the systematic difference at $T_{\mathrm{eff}}^{\mathrm{LSP} 3}>7000 \mathrm{~K}$, we expected that precisions of the KPCA temperatures are comparable to those of the LSP3 estimates.

The overall difference of surface gravity $\log g$ is $-0.03 \pm$ 0.21 dex for dwarf stars, whose KPCA values are estimated with the MILES training stars, and $-0.02 \pm 0.28$ dex for giant stars, whose KPCA values are estimated with the LAMOST-Kepler training stars. Though the dispersions seem not too big considering the low resolution of the LAMOST spectra, significant improvement has been achieved in the sense that the current results suffer much less systematic patterns. For the dwarf star sample, stars with LSP3 $\log g$ around 4.0 dex may have a wide range of $\log g$ values $(3.0-4.5$ dex $)$ in the KPCA results. This is because the LSP3 $\chi^{2}-$ based weighted-mean algorithm has artificially suppressed $\log g$ estimates due to the fact $\chi^{2}$ values are not sensitive enough to $\log g$ and that $\log g$ of the bulk dwarf/subgiant star templates covers only a limited range $(\sim 3.5-4.5 \mathrm{dex})$. As we increase the SNR cut of the sample to 100 , the mean difference becomes $-0.03 \pm 0.19$ dex, which changes minor respect to the $-0.03 \pm 0.21$ dex for a SNR cut of 20, indicating that the differences are dominated by systematics. For the giant star sample, clear patterns of $\log g$ also presented in the Figure. The presented patterns are consistent with those yielded by a direct comparison of the LSP3 weighted-mean $\log g$ with the asteroseismic values (cf. Fig. 6 of Ren et al. 2016). A similar exercise show that when we increase the SNR cut to 100 , the mean difference becomes $-0.02 \pm 0.26$ dex, again changes minor with

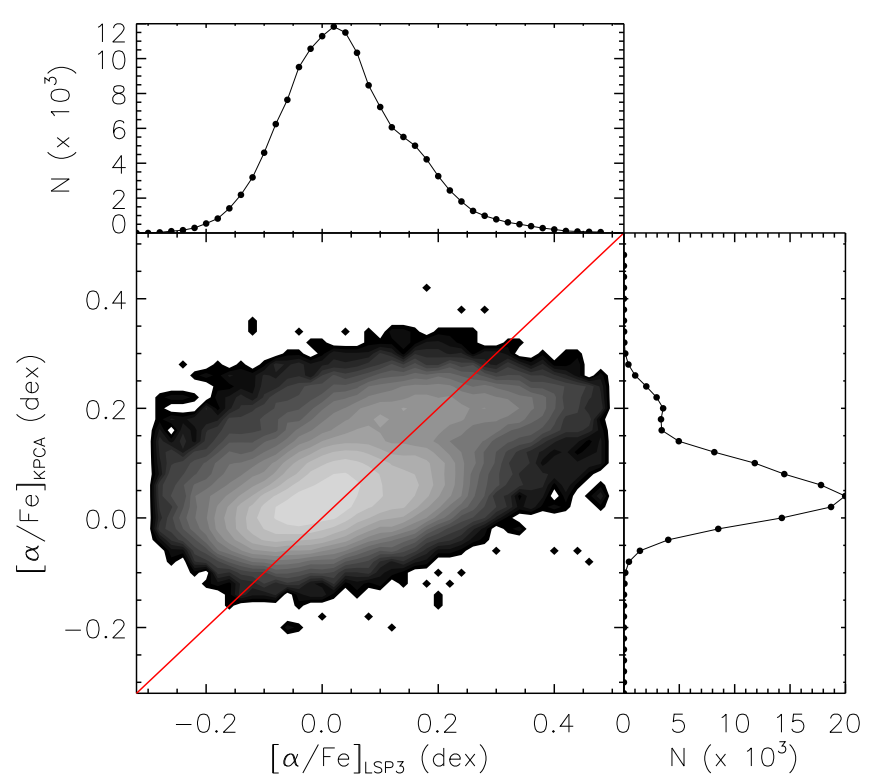

Figure 23. Comparison of the estimated $[\alpha / \mathrm{Fe}]$ with those derived with the LSP3 by a $\chi^{2}$-based template matching with synthetic spectral library for LSS-GAC giant stars. The side panels plot the 1-dimensional stellar number density distribution of both data sets.

respect to that of a SNR cut of 20, indicating that systematics dominate the differences.

Both metallicities estimated using the MILES stars and the LAMOST-APOGEE training sets are consistent well with the LSP3 estimates except for very metal-poor $([\mathrm{Fe} / \mathrm{H}]<-2.0 \mathrm{dex})$ stars or stars of super-solar metallicity. Note that here we have used the $[\mathrm{M} / \mathrm{H}]$ for metallicity estimated using the LAMOST-APOGEE training set, but we adopt the tab $[\mathrm{Fe} / \mathrm{H}]$ in the Figure for consistency. For $[\mathrm{Fe} / \mathrm{H}]$ estimated using the MILES training set, the overall difference respect to the LSP3 values is $-0.06 \pm 0.11$ dex. The -0.06 dex systematic offset is likely caused by an overestimate of the LSP3 values (Xiang et al. 2015a). At the metal-rich end, the current estimates are systematically higher than the LSP3 values. This is a natural consequence of the underestimation of the LSP3 $[\mathrm{Fe} / \mathrm{H}]$ due to the so-called 'boundary effects' of the LSP3 weighted-mean algorithm Xiang et al. (2015a), and one can see the sharp boundary in the Figure. At the metal-poor end, it is likely that both methods can not provide reliable $[\mathrm{Fe} / \mathrm{H}]$ estimates due to the lack of template (training) stars of $[\mathrm{Fe} / \mathrm{H}]<-2.5$ dex. For $[\mathrm{Fe} / \mathrm{H}]$ deduced with the LAMOST-APOGEE training set, the overall difference is $0.05 \pm 0.14$ dex. The 0.05 dex systematic offset is largely contributed by the underestimation of the LSP3 values at the metal-rich side due to the boundary effect of the LSP3 weightedmean algorithm. For giant stars with $[\mathrm{Fe} / \mathrm{H}]_{\mathrm{LSP} 3}<-2.0 \mathrm{dex}$, the KPCA estimates give a value between -1.0 and -1.8 dex. For these stars, it is probably that the KPCA values are overestimated due to a lack of sufficient LAMOST-APOGEE training stars with $[\mathrm{Fe} / \mathrm{H}]<-1.5$ dex.

We compare our $[\alpha / \mathrm{Fe}]$ estimates with those determined with a $\chi^{2}$-based template-matching method of LSP3 (Li et al. 2016). Fig. 23 shows the comparison for LSS-GAC giant stars with a spectral SNR higher than 30. The Figure shows that though there is a correlation between the two sets of measurements, their morphologies of distribution are quite different. The $[\alpha / \mathrm{Fe}]$ estimated in this 

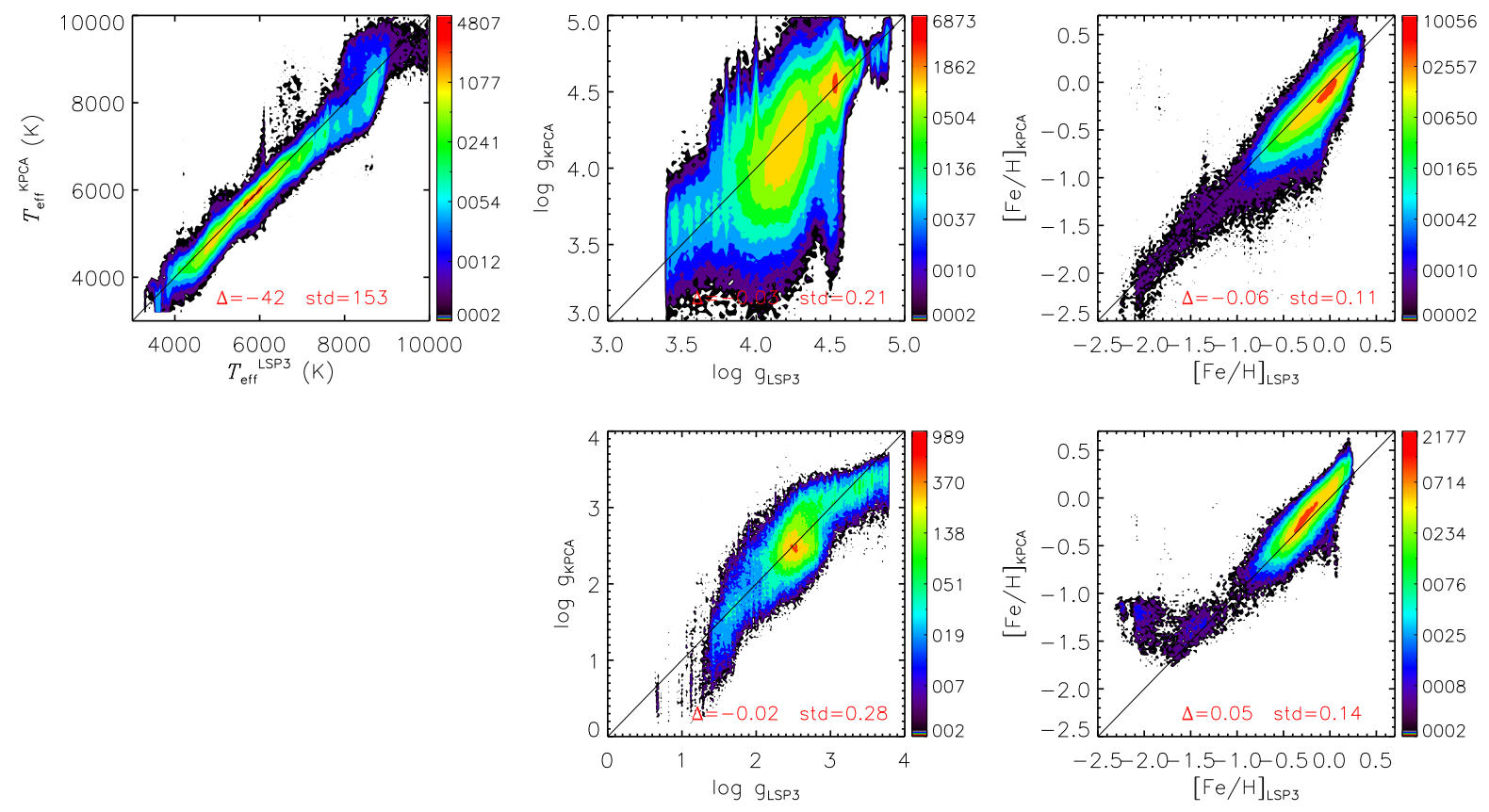

Figure 22. Comparison of the estimated parameters with those derived with the weighted-mean method of the LSP3 for the whole LSS-GAC star sample of spectral SNR higher than 20. Colours indicate stellar number density in logarithmic scale. The upper panels show results for parameters estimated with the MILES training stars, while the lower panels show results for $\log g$ estimated with the LAMOST-Kepler training set and [Fe/H] estimated with the LAMOST-APOGEE training set. A resistant estimate of the overall mean and standard deviation are marked in the plots.

work have a narrower distribution, with only a few stars having a value smaller than -0.1 dex or larger than $0.3 \mathrm{dex}$, while $[\alpha / \mathrm{Fe}]$ estimated with the $\chi^{2}$ method of LSP3 show a significantly wider distribution. Though our current estimates are not externally calibrated to standard data sets, which means that systematic bias could be hided in our results, it is no doubt that the difference of morphology are largely caused by the larger random errors $(0.08-0.1$ dex $)$ of $[\alpha / \mathrm{Fe}]$ derived with the $\chi^{2}$ method. We have done a test by assigning extra random errors of 0.05 dex into the KPCA results, and find that the distributions are much more resemble to each other, but systematic trend still remains. More examinations, especially calibration against high-resolution spectroscopic data sets, are necessary to further declare the accuracy of both the current estimates and the $\chi^{2}$ results. Nevertheless, it is remarkable that the $[\alpha / \mathrm{Fe}]$ and $[\alpha / \mathrm{M}]$ derived in this work have surprisingly high precisions, and the distribution of stars in the $[\mathrm{M} / \mathrm{H}]-[\alpha / \mathrm{M}]$ as well as the $[\mathrm{Fe} / \mathrm{H}]$ $-[\alpha / \mathrm{Fe}]$ plane is quite similar to those from high-resolution spectroscopy (e.g. Venn et al. 2004; Fuhrmann 2008; Holtzman et al. 2015; ;ordopatis et al. 2015).

\section{DISCUSSION}

As has mentioned, the current work is carried out with several main considerations: one is to extract 'weak' features that sensitive to parameters such as $\log g,[\mathrm{M} / \mathrm{H}],[\alpha / \mathrm{M}]$ and individual elemental abundances automatically from the LAMOST spectra with the KPCA algorithm, thus to have a precise determination of those parameters for the millions stars of the LAMOST survey. Another consideration is to use a regression algorithm for parameter estimation to avoid potential artifacts (systematic patterns) that caused by inadequacy of the weighted-mean algorithm of the LSP3. Since the coverage of the empirical spectral templates in the parameter space is limited, a weighted-mean algorithm inevitably causes systematics to parameters of stars located near or outside the boundary of the parameter space. We call such an effect the 'boundary effect' in Xiang et al. (2015a). In fact, because the empirical templates are not homogeneously distributed in the parameter space, stars located in the inner parameter space could also suffer systematics with the weighted-mean method if the weights toward individual templates are not properly assigned, which may cause artificial clustering of the resultant parameters. In addition, the current work has also, for the first time, determined absolute magnitudes and elemental abundances $[\mathrm{C} / \mathrm{H}]$ and $[\mathrm{N} / \mathrm{H}]$ from the LAMOST spectra.

The above detailed examinations have shown that the current method has achieved high precisions for the estimates of $T_{\mathrm{eff}}, \log g$, $\mathrm{M}_{V}$ and $\mathrm{M}_{K \mathrm{~s}},[\mathrm{Fe} / \mathrm{H}]([\mathrm{M} / \mathrm{H}]),[\alpha / \mathrm{Fe}]([\alpha / \mathrm{M}])$ as well as $[\mathrm{C} / \mathrm{H}]$ and $[\mathrm{N} / \mathrm{H}]$ for LAMOST stars. Those high precisions validate the good capabilities of the KPCA algorithm for deducing stellar parameters from the LAMOST spectra. Systematic patterns hided in results of the LSP3 weighted-mean algorithm, for instance, the boundary effects of $[\mathrm{Fe} / \mathrm{H}]$ in the metal-rich end, and the artificial suppression of $\log g$ for the dwarf/subgiant stars, have been largely reduced. Those improvements are expected to significantly promote our capability for classification of stellar population, estimation of stellar distance and age, as well as the address of many other scientific issues. However, we emphasize that because different training sets are used to estimate parameters for stars in different parameter ranges, some caution is needed when interpreting the results if mixed samples are used. More comments about this will be presented in the data release paper (Xiang et al. 2016, in preparation).

Nevertheless, there are still large space for further improvement of the parameter estimation. Sufficient stars with accurate, independent measurements of the stellar parameters that cover- 
ing enough volume in the parameter space are still needed for either the calibration of the derived parameters or the supplementation of our training sets. For $\log g$, the asteroseismic sample of dwarf/subgiant stars are limited to only a small range of both $T_{\text {eff }}(\sim 5400-6500 \mathrm{~K})$ and $[\mathrm{Fe} / \mathrm{H}]$ (dominated by stars of $[\mathrm{Fe} / \mathrm{H}]>-1.0 \mathrm{dex})$. The current $\log g$ estimates achieve a precision of 0.1 dex only for late type $\left(T_{\text {eff }}<6500 \mathrm{~K}\right)$ and metal-rich $([\mathrm{Fe} / \mathrm{H}]>-1.0$ dex $)$ stars, while for stars with higher temperature or lower metallicity, uncertainties in $\log g$ estimates are significantly larger. For $[\mathrm{Fe} / \mathrm{H}]$, both the MILES training stars and the high-resolution spectroscopy data sets adopted for the examination are limited to $[\mathrm{Fe} / \mathrm{H}]>-2.5 \mathrm{dex}$, and the number of stars with $[\mathrm{Fe} / \mathrm{H}]<-1.0 \mathrm{dex}$ is small. What's more, the high-resolution spectroscopy data sets are collected from various literatures, and are not uniformly calibrated. The LAMOST-Hipparcos test stars are also limited to metal-rich stars only, thus the estimated absolute magnitudes for metal-poor stars need to be further examined. Effects from binaries, double/multiple stars as well as variable stars on the determination of stellar atmospheric parameters and absolute magnitudes need to be further investigated systematically. The number of very metal-poor stars in the LAMOST-APOGEE training set is still quite few. As a result, our estimates of elemental abundances for stars of $[\mathrm{M} / \mathrm{H}]<-1.5 \mathrm{dex}$ are systematically overestimated. Similarly, because there are only very few stars with high $[\alpha / \mathrm{Fe}](>0.3 \mathrm{dex})$, in our training set, $[\alpha / \mathrm{Fe}]$ for such stars are probably estimated incorrectly. In addition, the APOGEE abundances are determined with $\chi^{2}$-based algorithms, they may suffer from systematic biases, which have propagated into our deduced elemental abundances via the LAMOST-APOGEE training set. Systematic differences have been found between the current results and $[\alpha / \mathrm{Fe}]$ yielded by the template-matching method of LSP3, while further studies are needed to understand those differences. Further calibration with independent measurements of elemental abundances from high-resolution spectroscopy seems to be necessary to reduce systematic errors in the results.

In future, parallax from Gaia (Perryman et al. 2001), combined with metallicity and elemental abundances from highresolution spectroscopy survey, e.g. the Gaia-ESO survey Gilmore et al. 2012), should be very helpful to build better training/calibration data sets for our parameter estimation from LAMOST spectra. More sophisticated algorithm and strategy to extract spectral features are also necessary to improve the measurements of elemental abundances as well as stellar atmospheric parameters.

\section{CONCLUSION}

We have estimated stellar atmospheric parameters ( $T_{\text {eff }}, \log g$, $[\mathrm{Fe} / \mathrm{H}])$, absolute magnitudes $\left(\mathrm{M}_{V}, \mathrm{M}_{K \mathrm{~s}}\right)$ and elemental abundances $([\mathrm{C} / \mathrm{H}],[\mathrm{N} / \mathrm{H}],[\alpha / \mathrm{M}],[\alpha / \mathrm{Fe}])$ from LAMOST spectra with a multivariate regression method based on kernel-based principal component analysis. Both internal and external examinations indicate that our method can yield stellar parameters from LAMOST spectra with a precision of $\sim 100 \mathrm{~K}$ for $T_{\text {eff }}, \sim 0.1$ dex for $\log g, 0.3-0.4 \mathrm{mag}$ for $\mathrm{M}_{V}$ and $\mathrm{M}_{K \mathrm{~s}}, \sim 0.1$ dex for $[\mathrm{M} / \mathrm{H}],[\mathrm{Fe} / \mathrm{H}]$, $[\mathrm{C} / \mathrm{H}]$ and $[\mathrm{N} / \mathrm{H}]$, and better than $0.05 \mathrm{dex}$ for $[\alpha / \mathrm{M}]([\alpha / \mathrm{Fe}])$, at a spectral SNR per pixel higher than 50 . Even at a spectral SNR of 20, the precisions are still quite satisfactory, with uncertainties of $\sim 150 \mathrm{~K}$ in $T_{\text {eff }}, 0.2 \mathrm{dex}$ in $\log g, \sim 0.5-0.6 \mathrm{mag}$ in $\mathrm{M}_{V}$ and $\mathrm{M}_{K \mathrm{~s}}, \sim 0.15 \mathrm{dex}$ in $[\mathrm{M} / \mathrm{H}],[\mathrm{Fe} / \mathrm{H}],[\mathrm{C} / \mathrm{H}]$ and $[\mathrm{N} / \mathrm{H}]$, and $\sim 0.06 \mathrm{dex}$ in $[\alpha / \mathrm{M}]([\alpha / \mathrm{Fe}])$. However, we note that those numbers are generally for relatively metal-rich stars $([\mathrm{Fe} / \mathrm{H}]>-1.5 \mathrm{dex})$, while pa- rameters for more metal-poor stars are much less precise/accurate, and need to be used with cautious. With this method, systematic patterns hided in the $\chi^{2}$-based weighted-mean stellar atmospheric parameters yielded by the LSP3 can be largely reduced.

Parameter errors for individual stars are detailedly assigned considering both errors induced by imperfections of the spectra and errors of the method, and the assigned errors are functions of the spectral SNR and stellar atmospheric parameters. Nevertheless, systematic offsets and biases may still exist in the results, especially in elemental abundances, due to a lack of external calibration against independent data sets with accurate parameter measurements, and simultaneously, with wide enough parameter coverage. Systematic biases in the estimated elemental abundances are expected to be $\sim 0.1-0.2$ dex.

The derived stellar atmospheric parameters, absolute magnitudes and elemental abundances for more than 1.4 million stars from the LSS-GAC, will be publicly available in the coming second release of value-added catalog of LSS-GAC (Xiang et al. 2016, in preparation). We also plan to release parameters of all stars targeted by the LAMOST Galactic surveys in due course.

Acknowledgments This work is supported by Joint Funds of the National Natural Science Foundation of China (Grant No. U1531244) and the National Key Basic Research Program of China 2014CB845700. Guoshoujing Telescope (the Large Sky Area Multi-Object Fiber Spectroscopic Telescope LAMOST) is a National Major Scientific Project built by the Chinese Academy of Sciences. Funding for the project has been provided by the National Development and Reform Commission. LAMOST is operated and managed by the National Astronomical Observatories, Chinese Academy of Sciences. M.S.X. thanks Prof. Chao Liu for helpful discussion.

\section{REFERENCES}

Allende Prieto C., Beers T. C., Wilhelm R., Newberg H. J., Rockosi C. M., Yanny B., Lee Y. S., 2006, ApJ, 636, 804

Anderson E., Francis C., 2012, Astronomy Letters, 38, 331

Bailer-Jones C. A. L. et al., 2013, A\&A, 559, A74

Bensby T., Feltzing S., Lundström I., 2003, A\&A, 410, 527

Bu Y., Pan J., 2015, MNRAS, 447, 256

Cenarro A. J. et al., 2007, MNRAS, 374, 664

Cui X.-Q. et al., 2012, Research in Astronomy and Astrophysics, 12,1197

De Cat P. et al., 2015, ApJS, 220, 19

Demarque P., Woo J.-H., Kim Y.-C., Yi S. K., 2004, ApJS, 155, 667

Deng L.-C. et al., 2012, Research in Astronomy and Astrophysics, 12,735

Dotter A., Chaboyer B., Jevremović D., Kostov V., Baron E., Ferguson J. W., 2008, ApJS, 178, 89

Falcón-Barroso J., Sánchez-Blázquez P., Vazdekis A., Ricciardelli E., Cardiel N., Cenarro A. J., Gorgas J., Peletier R. F., 2011, A\&A, 532, A95

Freeman K. C., 2012, in Astronomical Society of the Pacific Conference Series, Vol. 458, Galactic Archaeology: Near-Field Cosmology and the Formation of the Milky Way, Aoki W., Ishigaki M., Suda T., Tsujimoto T., Arimoto N., eds., p. 393

Fuhrmann K., 2008, MNRAS, 384, 173 
Gao H. et al., 2015, Research in Astronomy and Astrophysics, 15, 2204

García Pérez A. E. et al., 2016, AJ, 151, 144

Gilmore G. et al., 2012, The Messenger, 147, 25

Hekker S., Elsworth Y., Mosser B., Kallinger T., Basu S., Chaplin W. J., Stello D., 2013, A\&A, 556, A59

Henden A., Munari U., 2014, Contributions of the Astronomical Observatory Skalnate Pleso, 43, 518

Holtzman J. A. et al., 2015, AJ, 150, 148

Hou J. L., Zhong J., Chen L., Yu J. C., Liu C., Deng L. C., 2013, in IAU Symposium, Vol. 292, IAU Symposium, Wong T., Ott J., eds., pp. 105-105

Huang Y., Liu X.-W., Yuan H.-B., Xiang M.-S., Chen B.-Q., Zhang H.-W., 2015a, MNRAS, 454, 2863

Huang Y. et al., 2015b, Research in Astronomy and Astrophysics, 15,1240

Huber D. et al., 2014, ApJS, 211, 2

Jacobson H. R., Pilachowski C. A., Friel E. D., 2011, AJ, 142, 59

Jofré P. et al., 2015, A\&A, 582, A81

Koleva M., Prugniel P., Bouchard A., Wu Y., 2009, A\&A, 501, 1269

Kordopatis G. et al., 2013, AJ, 146, 134

Kordopatis G. et al., 2015, A\&A, 582, A122

Lee Y. S. et al., 2008a, AJ, 136, 2022

Lee Y. S. et al., 2008b, AJ, 136, 2050

Li J. et al., 2016, Research in Astronomy and Astrophysics, 16, 010

Li X., Lu Y., Comte G., Luo A., Zhao Y., Wang Y., 2015, ApJS, 218,3

Liu C. et al., 2015, ApJ, 807, 4

Liu X.-W. et al., 2014, in IAU Symposium, Vol. 298, IAU Symposium, Feltzing S., Zhao G., Walton N. A., Whitelock P., eds., pp. 310-321

Liu X.-W., Zhao G., Hou J.-L., 2015, Research in Astronomy and Astrophysics, 15, 1089

Lu Y., Li X., 2015, MNRAS, 452, 1394

Luo A.-L. et al., 2012, Research in Astronomy and Astrophysics, 12,1243

Luo A.-L. et al., 2015, Research in Astronomy and Astrophysics, 15,1095

Majewski S. R., Wilson J. C., Hearty F., Schiavon R. R., Skrutskie M. F., 2010, in IAU Symposium, Vol. 265, IAU Symposium, Cunha K., Spite M., Barbuy B., eds., pp. 480-481

Manteiga M., Ordóñez D., Dafonte C., Arcay B., 2010, PASP, 122,608

Müller K. R., Mika S., Rätsch G., Tsuda K., Schölkopf B., 2001, IEEE Transactions on Neural Networks, 12, 181

Ness M., Hogg D. W., Rix H.-W., Ho A. Y. Q., Zasowski G., 2015, ApJ, 808, 16

Perryman M. A. C. et al., 2001, A\&A, 369, 339

Perryman M. A. C. et al., 1997, A\&A, 323, L49

Prugniel P., Soubiran C., Koleva M., Le Borgne D., 2007, ArXiv Astrophysics e-prints, 0703658

Re Fiorentin P., Bailer-Jones C. A. L., Lee Y. S., Beers T. C., Sivarani T., Wilhelm R., Allende Prieto C., Norris J. E., 2007, A\&A, 467, 1373

Recio-Blanco A., Bijaoui A., de Laverny P., 2006, MNRAS, 370, 141

Recio-Blanco A. et al., 2016, A\&A, 585, A93

Ren J.-J. et al., 2016, Research in Astronomy and Astrophysics, 16,009
Richer H. B., Fahlman G. G., Rosvick J., Ibata R., 1998, ApJL, 504, L91

Salaris M., Weiss A., Percival S. M., 2004, A\&A, 414, 163

Sánchez-Blázquez P. et al., 2006, MNRAS, 371, 703

Schlegel D. J., Finkbeiner D. P., Davis M., 1998, ApJ, 500, 525

Schölpokf B., Smola A. J., Müller K. R., 1998, Neural Computation, 10, 1299

Skrutskie M. F. et al., 2006, AJ, 131, 1163

Soubiran C., Le Campion J.-F., Cayrel de Strobel G., Caillo A., 2010, A\&A, 515, A111

Steinmetz M. et al., 2006, AJ, 132, 1645

Venn K. A., Irwin M., Shetrone M. D., Tout C. A., Hill V., Tolstoy E., 2004, AJ, 128, 1177

Wang S.-G., Su D.-Q., Chu Y.-Q., Cui X., Wang Y.-N., 1996, Appl. Opt., 35, 5155

Wenger M. et al., 2000, A\&AS, 143, 9

Wu Y., Du B., Luo A., Zhao Y., Yuan H., 2014, in IAU Symposium, Vol. 306, Statistical Challenges in 21st Century Cosmology, Heavens A., Starck J.-L., Krone-Martins A., eds., pp. 340342

Wu Y. et al., 2011, Research in Astronomy and Astrophysics, 11, 924

Xiang M. S. et al., 2015a, MNRAS, 448, 822

Xiang M.-S. et al., 2015b, Research in Astronomy and Astrophysics, 15, 1209

Xiang M. S. et al., 2015c, MNRAS, 448, 90

Yang T., Li X., 2015, MNRAS, 452, 158

Yanny B. et al., 2009, AJ, 137, 4377

Yuan H.-B. et al., 2015, MNRAS, 448, 855

Yuan H. B., Liu X. W., Xiang M. S., 2013, MNRAS, 430, 2188

Zhang H.-H., Liu X.-W., Yuan H.-B., Zhao H.-B., Yao J.-S., Zhang H.-W., Xiang M.-S., Huang Y., 2014, Research in Astronomy and Astrophysics, 14, 456

Zhang J. N., Wu F. C., Luo A. L., Zhao Y. H., 2005, Acta Astronomica Sinica, 46, 406

Zhao G., Zhao Y.-H., Chu Y.-Q., Jing Y.-P., Deng L.-C., 2012, Research in Astronomy and Astrophysics, 12, 723

Zucker D. B., de Silva G., Freeman K., Bland-Hawthorn J., Hermes Team, 2012, in Astronomical Society of the Pacific Conference Series, Vol. 458, Galactic Archaeology: Near-Field Cosmology and the Formation of the Milky Way, Aoki W., Ishigaki M., Suda T., Tsujimoto T., Arimoto N., eds., p. 421

Zwitter T. et al., 2008, AJ, 136, 421 